Pacific

Journal of

Mathematics

\title{
AN END-TO-END CONSTRUCTION FOR COMPACT CONSTANT MEAN CURVATURE SURFACES
}

MOHAMEd JLELI AND FRANK PACARD 


\title{
AN END-TO-END CONSTRUCTION FOR COMPACT CONSTANT MEAN CURVATURE SURFACES
}

\author{
MOHAMED JLELI AND FRANK PACARD
}

\begin{abstract}
We provide a construction for new compact surfaces of constant mean curvature of genus 3 and higher, based on tools developed for the understanding of complete noncompact constant mean curvature surfaces and the endto-end construction developed by J. Ratskin to connect (and produce) complete noncompact constant mean curvature surfaces along their ends.
\end{abstract}

\section{Introduction}

The theory of constant mean curvature surfaces in Euclidean space has been the object of intensive study in the past years. In the case of complete noncompact constant mean curvature surfaces, the moduli space of such surfaces is now fairly well understood (at least in the genus 0 case); see [Kusner et al. 1996; GroßeBrauckmann 2000; 2003]. Many techniques have been developed to produce examples of such surfaces [Große-Brauckmann 1993; Kapouleas 1990; Mazzeo and Pacard 2001; Mazzeo et al. 2005].

By contrast, the set of compact constant mean curvature surfaces is not so well understood. H. Wente [1986] was the first to construct genus 1 constant mean curvature surfaces. These genus 1 surfaces were then thoughtfully studied by U. Pinkall and I. Sterling [1989]. Examples of compact constant mean curvature surfaces of higher genus are due to N. Kapouleas. In the genus 2 case [Kapouleas 1995], these surfaces are obtained by "fusing" Wente tori, while for genus greater than 2 they are obtained by connecting together many mutually tangent unit spheres, using small catenoid necks [Kapouleas 1991].

In this paper we propose to explain how current knowledge on the set of complete noncompact constant mean curvature surfaces can be exploited to produce new examples of compact constant mean curvature surfaces of genus 3 and higher.

Our construction is based on tools that have been developed for the understanding of complete noncompact constant mean curvature surfaces. It can be described as follows:

MSC2000: 53A10, 53C42, 53C21.

Keywords: compact constant mean curvature surfaces. 
(1) Since the first construction by N. Kapouleas [1990], many constructions of complete noncompact constant mean curvature surfaces have been developed; see the references cited in the first paragraph. These constructions provide an important source of examples of complete noncompact constant mean curvature surfaces the geometry of whose ends is partially prescribed.

(2) Most of these constructions are flexible and one can arrange it so that the ends of these surfaces can be plugged together to produce sequences (indexed by a discrete parameter $n \in \mathbb{N}$ ) of compact surfaces having mean curvature 1 except in finitely many annular regions, where their mean curvature can be estimated by $1+O\left(e^{-\gamma n}\right)$ for some $\gamma>0$. This is essentially the end-to-end construction which was developed by J. Ratzkin [2001] to connect (and produce) complete noncompact constant mean curvature surfaces along their ends.

(3) Next we study the mapping properties of the bounded operator about this surface of almost constant mean curvature. To perform this analysis, we rely on the fact that parametrices for the Jacobi operators on each complete noncompact summand have been obtained in the moduli space theory developed by R. Kusner, R. Mazzeo and D. Pollack [Kusner et al. 1996]. We explain how these can be glued together. This construction requires a precise understanding of the set of Jacobi fields on each summand.

(4) Finally, we use a standard perturbation argument to produce sequences of compact constant mean curvature surfaces of arbitrary genus $\geq 3$.

We believe that the main advantage of our construction over Kapouleas' is that it is technically simple (once the results on complete noncompact surfaces mentioned above are understood!). This parallels the fact that the end-to-end construction of J. Ratzkin is simpler than the previous constructions of complete noncompact surfaces. We obtain a very precise description of the surfaces we produce: the perturbation of the approximate surface is an exponentially decreasing function of the diameter of the surface constructed. In particular, our construction sheds light on the structure of the set of compact constant mean curvature surfaces, showing that these surfaces are isolated. Though this is probably a minor point, the example of compact constant mean curvature surfaces we obtain are geometrically different from the one obtained by Kapouleas; roughly speaking, all his surfaces are close to sequences of unit spheres linked by small catenoids, and hence have small injectivity radius, while our examples need not have small necks, and hence have injectivity radius uniformly bounded away from below.

A possibly more important issue is that our construction points to interesting directions toward which the theory of complete noncompact constant mean curvature surfaces should be developed to understand the set of compact constant mean curvature surfaces. In previous constructions some properties of complete 
noncompact constant mean curvature surfaces have been neglected and they turn out to be extremely important. This is the case, for example, with the notion of nondegeneracy and the notion of a regular end (both turn out to be also important in the construction of J. Ratzkin).

Finally, our construction generalizes to any dimension [Jleli 2004].

Although our method can be applied to produce nonsymmetric surfaces, a complete description of the set of compact surfaces is far beyond our understanding. This is why we have chosen not to look for the utmost generality but to focus on the construction of symmetric surfaces, thus keeping notation as simple as possible. We will therefore explain how to construct constant mean curvature surfaces of arbitrary genus $\geq 3$ having dihedral symmetry. More precisely, we show that there exists a nonempty open interval $O$ and a smooth bounded function $\Xi: O \rightarrow \mathbb{R}$ such that any solution $(\zeta, n) \in O \times \mathbb{N}$ of the relation

$$
\Xi(\zeta)+n \zeta \in \mathbb{N}
$$

gives rise to a constant mean curvature surface which has genus $k$ and is invariant under the action of the group

$$
G_{k}:=\left\{R_{2 \pi j / k}: j \in \mathbb{Z}\right\},
$$

where $R_{\theta}$ is the rotation of angle $\theta$ in the $x_{1}, x_{2}$ plane, as well as under the action of the symmetries with respect to the plane $x_{1}=0$ and the plane $x_{3}=0$. Moreover, distinct solutions of (1-1) give rise to geometrically distinct constant mean curvature surfaces and these surfaces are isolated in the set of constant mean curvature surfaces that are invariant under these symmetries.

In Sections 2 and 3 we recall facts about the set of Delaunay surfaces and moduli space theory. Then, in Section 4 we describe families of constant mean curvature surfaces with finitely many ends. The geometric construction is the content of Section 5. In Section 6 we develop the linear analysis; in particular we explain how the different parametrices on each summand can be glued together. The perturbation argument and the main result of the paper are explained in Section 7.

\section{Delaunay surfaces}

In this section we recall some well known facts concerning the family of Delaunay surfaces $D_{\tau}$, which are rotationally invariant constant mean curvature surfaces in $\mathbb{R}^{3}$ [Delaunay 1841]. We refer to [Mazzeo and Pacard 2001] for further details.

Isothermal parametrization. Delaunay surfaces can be parametrized, in isothermal coordinates, by

$$
X_{\tau}(s, \theta)=\frac{1}{2}\left(\tau e^{\sigma(s)} \cos \theta, \tau e^{\sigma(s)} \sin \theta, \kappa(s)\right),
$$


where $(s, \theta) \in \mathbb{R} \times S^{1}$ and where the functions $\sigma$ and $\kappa$ are described as follows: For any $\tau \in(0,1]$, the function $\sigma$ is defined to be the unique smooth nonconstant solution of the ordinary differential equation

$$
\left(\partial_{S} \sigma\right)^{2}+\tau^{2} \cosh ^{2} \sigma=1, \quad \partial_{s} \sigma(0)=0, \quad \sigma(0)<0,
$$

while, for any $\tau \in(-\infty, 0)$, the function $\sigma$ is defined to be the unique smooth nonconstant solution of the ordinary differential equation

$$
\left(\partial_{s} \sigma\right)^{2}+\tau^{2} \sinh ^{2} \sigma=1, \quad \partial_{s} \sigma(0)=0, \quad \sigma(0)<0 .
$$

Again, the definition of $\kappa$ differs according to the sign of $\tau$. When $\tau \in(0,1]$, we define the function $\kappa$ by

$$
\partial_{s} \kappa=\tau^{2} e^{\sigma} \cosh \sigma, \quad \kappa(0)=0,
$$

while when $\tau<0$, we define the function $\kappa$ by

$$
\partial_{s} \kappa=\tau^{2} e^{\sigma} \sinh \sigma, \quad \kappa(0)=0 .
$$

Observe that, when $\tau>0, \kappa$ is monotone increasing and hence $X_{\tau}$ is an embedding, whereas, when $\tau<0$, this is no longer true and the surface parametrized by $X_{\tau}$ is only immersed. The embedded and immersed Delaunay surfaces $D_{\tau}$ are called unduloids and nodoids, respectively. The parameter $\tau$ will be referred to as the Delaunay parameter.

We fix a positively oriented orthonormal basis $\left(\vec{e}_{1}, \vec{e}_{2}, \vec{e}_{3}\right)$ of $\mathbb{R}^{3}$.

Definition 2.1. Given a vector $\vec{a}$, with $|\vec{a}|=1$, the surface $D_{\tau}^{\vec{a}}$ is defined to be the image of the surface $D_{\tau}$ by a rotation which sends $\vec{e}_{3}$ to $\vec{a}$. We denote by $X_{\tau}^{\vec{a}}$ its parametrization. The unit normal vector field compatible with the orientation of $D_{\tau}^{\vec{a}}$ will be denoted by $\overrightarrow{N_{\tau}} \vec{a}$.

In particular, the axis of $D_{\tau}^{\vec{a}}$ is the line directed by $\vec{a}$ passing through the origin and $D_{\tau}^{\vec{a}}$ is invariant under the symmetry with respect to the plane whose normal is $\vec{a}$. Granted this notation, the Delaunay surface $D_{\tau}$ described in (2-1) is equal to $D_{\tau}^{\vec{e}_{3}}$ and we will simply write $\vec{N}_{\tau}$ instead of $\vec{N}_{\tau}^{\vec{e}_{3}}$.

Definition 2.2. Given a vector $\vec{b}$, the surface $D_{\tau}^{\vec{a}}+\vec{b}$ is defined to be the image of the surface $D_{\tau}^{\vec{a}}$ by the translation of vector $\vec{b}$. It is parametrized by $X_{\tau}^{\vec{a}}+\vec{b}$.

It is easy to check that the functions $\sigma$ are periodic and this translates into the fact that Delaunay surfaces are invariant under the action of a one-parameter discrete group of (vertical) translations. When $\tau \neq 1$, we define $s_{\tau}$ to be equal to half the least period of $\sigma$ and the half of the least vertical period of the Delaunay surface $D_{\tau}$ will be denoted by $T_{\tau}$. It is given by

$$
T_{\tau}:=\frac{1}{4} \kappa\left(2 s_{\tau}\right) .
$$


Warning. We emphasize that, with the conventions above, $2 s_{\tau}$ is equal to the least period of the function $\sigma$ and $2 T_{\tau}$ is the least vertical period of $D_{\tau}$. Hence we have the identity

$$
D_{\tau}^{\vec{a}}+2 T_{\tau} \vec{a}=D_{\tau}^{\vec{a}} .
$$

Lemma 2.3. For all $\tau \in(-\infty, 0) \cup(0,1]$, we have $\partial_{\tau} T_{\tau}>0$.

Proof. Observe that $\partial_{s} \sigma>0$ on $\left(0, s_{\tau}\right)$ and $\partial_{s} \sigma<0$ on $\left(s_{\tau}, 2 s_{\tau}\right)$. Hence, for $s \in\left(0, s_{\tau}\right)$, we can use $\sigma$ as a change of variable and express $\kappa$ as a function of $\sigma \in\left(-\sigma_{*}, \sigma_{*}\right)$ where $\sigma_{*}>0$ satisfies $\tau^{2} \cosh ^{2} \sigma_{*}=1$ when $\tau \in(0,1]$ and $\sigma_{*}>0$ satisfies $\tau^{2} \sinh ^{2} \sigma_{*}=1$ when $\tau<0$.

When $\tau<0$, we get

$$
2 T_{\tau}=\int_{-\sigma_{*}}^{\sigma_{*}} \frac{\tau^{2} e^{\sigma} \sinh \sigma}{\sqrt{1-\tau^{2} \sinh ^{2} \sigma}} d \sigma=\int_{-\sigma_{*}}^{\sigma_{*}} \frac{\tau^{2} \sinh ^{2} \sigma}{\sqrt{1-\tau^{2} \sinh ^{2} \sigma}} d \sigma .
$$

Performing the change of variable $\tau \sinh \sigma=\sin x$ we conclude that

$$
2 T_{\tau}=\int_{-\pi / 2}^{\pi / 2} \frac{\sin ^{2} x}{\sqrt{\tau^{2}+\sin ^{2} x}} d x,
$$

which clearly implies that $\partial_{\tau} T_{\tau}>0$ when $\tau<0$.

When $\tau>0$, we have

$$
2 T_{\tau}=\int_{-\sigma_{*}}^{\sigma_{*}} \frac{\tau^{2} e^{\sigma} \cosh \sigma}{\sqrt{1-\tau^{2} \cosh ^{2} \sigma}} d \sigma=\int_{-\sigma_{*}}^{\sigma_{*}} \frac{\tau^{2} \cosh ^{2} \sigma}{\sqrt{1-\tau^{2} \cosh ^{2} \sigma}} d \sigma .
$$

Performing the change of variable $\tau \sinh \sigma=\sqrt{1-\tau^{2}} \sin x$ we conclude that

$$
2 T_{\tau}=\int_{-\pi / 2}^{\pi / 2} \sqrt{1-\left(1-\tau^{2}\right) \cos ^{2} x} d x
$$

which again implies that $\partial_{\tau} T_{\tau}>0$.

The Jacobi operator. Assume that $\Sigma$ is a constant mean curvature surface. Any surface which is close enough to $\Sigma$ may be represented as a normal graph over $\Sigma$

$$
\Sigma_{w}=\{x+w(x) \vec{N}(x): x \in \Sigma\},
$$

where $\vec{N}$ is the unit normal vector field compatible with the orientation of $\Sigma$ and $w$ is a (small) function. The mean curvature of $\Sigma_{w}$ is denoted by $H(w)$. By definition, the Jacobi operator about $\Sigma$ is the differential of the mapping $w \mapsto 2 H(w)$ at $w=0$. It is given in terms of the geometric data of $\Sigma$ by

$$
\mathscr{L}_{\Sigma}:=\Delta_{\Sigma}+\left|A_{\Sigma}\right|^{2}
$$


where $\Delta_{\Sigma}$ is the Laplace-Beltrami operator on $\Sigma$ and $\left|A_{\Sigma}\right|^{2}$ is the square of the norm of the second fundamental form on $\Sigma$. A solution $w$ of the homogeneous problem $\mathscr{L}_{\Sigma} w=0$ is called a Jacobi field.

In the case where $\Sigma$ is the Delaunay surface $D_{\tau}$, the Jacobi operator will be denoted by $L_{\tau}$. In terms of the isothermal parametrization given in the previous paragraph, it is given by

$$
L_{\tau}=\frac{4}{\tau^{2} e^{2 \sigma}}\left(\partial_{s}^{2}+\partial_{\theta}^{2}+\tau^{2} \cosh (2 \sigma)\right) .
$$

For the sake of simplicity, we shall now assume that $\tau \neq 1$, namely that $D_{\tau}$ is not a cylinder. There is no loss of generality in doing so since our construction, which is based on the end-to-end construction, does not work for surfaces which have ends asymptotic to cylinders. Some Jacobi fields can be easily described since they correspond to explicit geometric deformations of Delaunay surfaces [Mazzeo and Pacard 2001]. We briefly describe these now since they will play a key role in the subsequent analysis.

The Jacobi fields corresponding to infinitesimal translations of $D_{\tau}$ will be denoted by $\Phi_{\tau}^{T, \vec{e}}$, where $|\vec{e}|=1$ (the indices $T, \vec{e}$ should remind the reader that this Jacobi field is associated to the "translation" of vector $\vec{e}$ ). They are obtained by projecting the constant vector field $\vec{e}$ over the normal vector field $\vec{N}_{\tau}$ about $D_{\tau}$. Hence

$$
\Phi_{\tau}^{T, \vec{e}}:=\vec{e} \cdot \vec{N}_{\tau} .
$$

It is geometrically obvious that there are three linearly independent such Jacobi fields (this is where we use the fact that $\tau \neq 1$ and hence that $D_{\tau}$ is not a cylinder). These Jacobi fields are periodic in the $s$ variable, hence they are bounded. Observe that $\Phi_{\tau}^{T, \vec{e}_{3}}$ only depends on $s$ while $\Phi_{\tau}^{T, \vec{e}_{1}}$ and $\Phi_{\tau}^{T, \vec{e}_{2}}$ depend on both $s$ and $\theta$.

The two Jacobi fields corresponding to infinitesimal rotations of the axis of $D_{\tau}$ will be denoted by $\Phi_{\tau}^{R, \vec{e}}$, where $|\vec{e}|=1$ and $\vec{e} \cdot \vec{e}_{3}=0$ (the indices $R, \vec{e}$ should remind the reader that this Jacobi field is associated to the "rotation" of the axis in the direction of the vector $\vec{e}$ ). They are obtained by projecting the Killing vector fields

$$
\vec{x} \mapsto(\vec{x} \cdot \vec{e}) \vec{e}_{3}-\left(\vec{x} \cdot \vec{e}_{3}\right) \vec{e}
$$

over the normal vector field $\vec{N}_{\tau}$; thus

$$
\Phi_{\tau}^{R, \vec{e}}:=(\vec{x} \cdot \vec{e}) \vec{e}_{3} \cdot \vec{N}_{\tau}-\left(\vec{x} \cdot \vec{e}_{3}\right) \vec{e} \cdot \vec{N}_{\tau}
$$

It is geometrically obvious that there are two linearly independent such Jacobi fields. This time, these Jacobi fields depend on both $s$ and $\theta$ and they "grow linearly in $s "$ as $|s|$ tends to $+\infty$; that is, there exists a constant $c>0$ (depending on $\tau$ ) 
such that

$$
c \leq \limsup _{|s| \rightarrow+\infty} \sup _{\theta \in S^{1}}\left((1+|s|)^{-1}\left|\Phi_{\tau}^{R, \vec{e}}\right|\right) .
$$

So far all the Jacobi fields we have defined can be explicitly written in terms of the function $\sigma$ and its derivatives [Mazzeo and Pacard 2001], even though we will not need these expressions. There is a last Jacobi field, whose geometric meaning is obvious but whose analytical expression is more intricate, which corresponds to the one-parameter family $D_{\tau}$ obtained by varying the Delaunay parameter $\tau$. This last Jacobi field will be denoted by $\Phi_{\tau}^{D}:=\partial_{\tau} X_{\tau} \cdot \vec{N}_{\tau}$ (the index $D$ should remind the reader that this Jacobi field is associated to the change of the Delaunay parameter). Observe that this Jacobi field only depends on $s$ since $D_{\tau}$ are surfaces of revolution.

That $\partial_{\tau} T_{\tau}$ is nonzero when $\tau \neq 1$ implies that this Jacobi field is again "linearly growing in $s$ " as $|s|$ tends to $+\infty$ in the sense above. Indeed, we have the identity

$$
X_{\tau}\left(\cdot+2 s_{\tau}, \cdot\right)=X_{\tau}+2 T_{\tau} \vec{e}_{3} .
$$

Differentiation with respect to $\tau$ yields

$$
2 \partial_{\tau} s_{\tau} \partial_{s} X_{\tau}\left(\cdot+2 s_{\tau}, \cdot\right)+\partial_{\tau} X_{\tau}\left(\cdot+2 s_{\tau}, \cdot\right)=\partial_{\tau} X_{\tau}+2 \partial_{\tau} T_{\tau} \vec{e}_{3} .
$$

Taking the scalar product with $\vec{N}_{\tau}$ we find

$$
\Phi_{\tau}^{D}\left(\cdot+2 s_{\tau}\right)=\Phi_{\tau}^{D}+2 \partial_{\tau} T_{\tau} \Phi_{\tau}^{T, \vec{e}_{3}},
$$

which clearly shows that $\Phi_{\tau}^{D}$ grows "linearly" in $s$.

The Jacobi operator $L_{\tau}$ being invariant under the action of rotations in $S^{1}$, we can perform the eigenfunction decomposition of any function $(s, \theta) \mapsto w(s, \theta)$ in the $\theta$ variable and the analysis of $L_{\tau}$ reduces to the analysis of the sequence of operators

$$
L_{\tau, j}:=\frac{4}{\tau^{2} e^{2 \sigma}}\left(\partial_{s}^{2}+\tau^{2} \cosh (2 \sigma)-j^{2}\right),
$$

for $j \in \mathbb{N}$. The potential in $L_{\tau, j}$ being periodic of period $s_{\tau}$ (observe that $\cosh (2 \sigma)$ is $s_{\tau}$-periodic since $\sigma$ is $2 s_{\tau}$-periodic and odd), it follows from Bloch wave theory [Mazzeo et al. 2005] that either

(i) the homogeneous problem $L_{\tau, j} w=0$ has two independent solutions $w^{ \pm}$(depending on $\tau$ and $j$ ) such that

$$
w^{ \pm}\left(s+s_{\tau}\right)=e^{ \pm \zeta_{\tau, j} s_{\tau}} w^{ \pm}(s)
$$

for some complex number $\zeta_{\tau, j}$, with $\Re \zeta_{\tau, j} \geq 0$, or

(ii) the homogeneous problem $L_{\tau, j} w=0$ has one periodic solution and one "linearly growing" solution; in this case, we set $\zeta_{\tau, j}:=0$. 
For each $\tau$ and $j$, we define the indicial roots associated to the operator $L_{\tau, j}$ to be the real numbers $\pm \gamma_{\tau, j}$ where

$$
\gamma_{\tau, j}:=\operatorname{Re} \zeta_{\tau, j} \geq 0
$$

It is proved in [Mazzeo et al. 2005] that:

Proposition 2.4. The indicial roots of $L_{\tau}$ enjoy the following properties:

(1) For any $\tau \in(-\infty, 0) \cup(0,1], \gamma_{\tau, 0}=\gamma_{\tau, 1}=0$.

(2) There exists $\tau_{*}<0$ such that, for all $j \geq 2$ and $\tau \in\left(\tau_{*}, 0\right) \cup(0,1], \gamma_{\tau, j}>0$.

The first property is a consequence of the fact that the Jacobi fields $\Phi_{\tau}^{D}, \Phi_{\tau}^{T, \vec{e}}$ and $\Phi_{\tau}^{R, \vec{e}}$ are either bounded or linearly growing, hence case (ii) always arises when $j=0$ or $j=1$. The second property follows from the maximum principle. Indeed, the potential $j^{2}-\tau^{2} \cosh (2 \sigma)$ which appears in $L_{\tau, j}$ can be seen to be bounded from below by a positive constant, when $j \geq 2$ and $\tau$ is negative close enough to 0 or positive (simply use the equation satisfied by $\sigma$ to obtain an upper bound for $\tau^{2} \cosh (2 \sigma)$ ). Therefore, when $j \geq 2$ and $\tau$ is not too far away from 0 , the maximum principle holds for $L_{\tau, j}$ and the existence of solutions of the homogeneous problem $L_{\tau, j} w=0$ which either blows up exponentially or decays exponentially at $\infty$ follows at once from the construction of barrier functions of the form $s \mapsto e^{\delta s}$.

\section{Moduli space theory}

We now briefly describe the moduli space theory for $k$-ended complete noncompact constant mean curvature surfaces as developed in [Kusner et al. 1996] and extended in [Mazzeo et al. 2005]. We define $\mathcal{M}_{g, k}^{\tau_{*}}$ to be the set of complete, noncompact constant mean curvature surfaces which have genus $g$ and $k$ ends asymptotic to Delaunay surfaces whose Delaunay parameter belongs to $\left(\tau_{*}, 0\right) \cup(0,1]$. Observe that we do not mod out by the group of rigid motions.

Warning. From now on, we assume that all Delaunay parameters we consider belong to $\left(\tau_{*}, 0\right) \cup(0,1]$, where $\tau_{*}$ is the constant defined in Proposition 2.4.

We can decompose each surface $\Sigma \in \mathcal{M}_{g, k}^{\tau_{*}}$ into overlapping connected pieces: a compact piece $K$ and the ends $E_{\ell}$, for $\ell=1, \ldots, k$. In addition, we require that each $K \cap E_{\ell}$ is homeomorphic to an annulus $[0,1] \times S^{1}$ and that, for each $\ell$, the end $E_{\ell}$ is parametrized by

$$
Y_{\ell}:=X_{\tau_{\ell}}^{\vec{a}_{\ell}}+w_{\ell} \vec{N}_{\tau_{\ell}}^{\vec{a}_{\ell}}+\vec{b}_{\ell},
$$

for $(s, \theta) \in[0,+\infty) \times S^{1}$. Here, we have chosen isothermal coordinates $(s, \theta)$ for the Delaunay surface $D_{\tau_{\ell}}^{\vec{a}_{\ell}}+\vec{b}_{\ell}$ on which $E_{\ell}$ is modeled. The fact that we have 
assumed that the end $E_{\ell}$ is asymptotic to $D_{\tau_{\ell}}^{\vec{a}_{\ell}}+\vec{b}_{\ell}$ means precisely that the function $w_{\ell}$ is exponentially decreasing. To be more specific, we need:

Definition 3.1. Given $r \in \mathbb{N}, \alpha \in(0,1)$ and $\mu \in \mathbb{R}$, the space $\underset{\mathscr{E}}{\mathscr{C}, \alpha}\left([0,+\infty) \times S^{1}\right)$ is the space of functions $v \in \mathscr{C}_{\text {loc }}^{r, \alpha}\left([0,+\infty) \times S^{1}\right)$ for which

$$
\|v\|_{\mathscr{C}_{\mu}^{r, \alpha}\left([0,+\infty) \times S^{1}\right)}:=\sup _{s \geq 0} e^{-\mu s}|v|_{\mathscr{C}^{r, \alpha}\left([s, s+1] \times S^{1}\right)}
$$

is finite. Here $|\cdot|_{\mathscr{G}^{r, \alpha}(\Omega)}$ denotes the usual Hölder norm in the set $\Omega$.

Granted this definition, it is known that the function $w_{\ell}$ defined in (3-1) satisfies

$$
w_{\ell} \in \mathscr{E}_{-\gamma_{\tau_{\ell}, 2}^{2, \alpha}}^{2}\left([0, \infty) \times S^{1}\right) .
$$

In other words the rate of decay of the function $w_{\ell}$ is dictated by the indicial root $\gamma_{\tau_{\ell}, 2}$. We refer to [Mazzeo and Pacard 2001] for a proof of this fact. The moduli space theory is based on the definition of weighted spaces on $\Sigma$ :

Definition 3.2. For $r \in \mathbb{N}, \alpha \in(0,1)$ and $\mu \in \mathbb{R}$, let $\mathscr{D}_{\mu}^{r, \alpha}(\Sigma)$ be the space of functions $v \in \mathscr{C}^{r, \alpha}(\Sigma)$ for which

$$
\|v\|_{\mathscr{D}_{\mu}^{r, \alpha}}^{r}:=\left.\left\|\left.v\right|_{K}\right\|\left\|_{\mathscr{C}^{r, \alpha}}+\sum_{\ell=1}^{k}\right\| v\right|_{E_{\ell}} \circ Y_{\ell} \|_{\mathscr{C}_{\mu}^{r, \alpha}\left([0,+\infty) \times S^{1}\right)}
$$

is finite.

Definition 3.3. The surface $\Sigma \in \mathcal{M}_{g, k}^{\tau_{*}}$ is said to be nondegenerate if

$$
\mathscr{L}_{\Sigma}: \mathscr{D}_{\mu}^{2, \alpha}(\Sigma) \rightarrow \mathscr{D}_{\mu}^{0, \alpha}(\Sigma)
$$

is injective for all $\mu<0$.

Following the analysis of the Jacobi fields we did above (starting on page 85) and using the parametrization (3-1) together with (3-2), it is easy to see that, on each end $E_{\ell}$ of $\Sigma$, there exists 5 (globally defined on $\Sigma$ ) independent Jacobi fields $\Phi_{E_{\ell}}^{T, \vec{e}}$ and $\Phi_{E_{\ell}}^{R, \vec{e}^{\prime}}$ which satisfy

$$
\begin{gathered}
\left.\Phi_{E_{\ell}}^{T, \vec{e}} \circ Y_{\ell}-\Phi_{\tau_{\ell}}^{T, \vec{e}} \in \mathscr{E}_{-\gamma_{\tau_{\ell}, 2}^{2, \alpha}}^{2,}\left([0,+\infty) \times S^{1}\right)\right), \\
\left.\Phi_{E_{\ell}}^{R, \vec{e}^{\prime}} \circ Y_{\ell}-\Phi_{\tau_{\ell}}^{R, \vec{e}^{\prime}} \in \mathscr{E}_{-\gamma_{\tau_{\ell}, 2}}^{2, \alpha}\left([0,+\infty) \times S^{1}\right)\right),
\end{gathered}
$$

where $|\vec{e}|=1,\left|\vec{e}^{\prime}\right|=1$ and $\vec{e}^{\prime} \cdot \vec{a}_{\ell}=0$.

The existence of a sixth Jacobi field $\Phi_{E_{\ell}}^{D}$ defined on $E_{\ell}$ and asymptotic to $\Phi_{\tau_{\ell}}^{D}$ is not a trivial fact and follows from a perturbation argument [Mazzeo and Pacard 2001]. Since $E_{\ell}$ is a graph over a Delaunay surface for some exponentially decaying function, such a Jacobi field can be constructed by perturbing $\Phi_{\tau}^{D}$, looking for a function $w$ for which $\Phi_{E_{\ell}}^{D}=\Phi_{\tau_{\ell}}^{D}+w$ satisfies $\mathscr{L}_{\Sigma} \Phi_{E_{\ell}}^{D}=0$ on $E_{\ell}$. In general, 
this Jacobi field is only defined on $E_{\ell}$ (eventually away from a compact set in $E_{\ell}$ ) and is not globally defined on $\Sigma$. This motivates the notion of a regular end:

Definition 3.4. The end $E_{\ell}$ of $\Sigma$ is said to be regular if there exists a Jacobi field $\Phi_{E_{\ell}}^{D}$, globally defined on $\Sigma$, satisfying

$$
\left.\Phi_{E_{\ell}}^{D} \circ Y_{\ell}-\Phi_{\tau_{\ell}}^{D} \in \mathscr{E}_{\mu_{0}}^{2, \alpha}\left([0,+\infty) \times S^{1}\right)\right),
$$

for all $\mu_{0} \in\left(-\gamma_{\tau_{\ell}, 2}, 0\right)$.

For example, such a globally defined Jacobi field can be obtained starting from a one-parameter family of constant mean curvature surfaces $\Sigma(\varepsilon) \in \mathcal{M}_{g, k}^{\tau_{*}}$, for $\varepsilon \in$ $\left(-\varepsilon_{0}, \varepsilon_{0}\right)$, which are close to $\Sigma$ (in a suitable sense), satisfy $\Sigma(0)=\Sigma$ and whose $\ell$-th end $E_{\ell}(\varepsilon)$ is asymptotic to a Delaunay surface of parameter $\tau_{\ell}+\varepsilon$. Writing $\Sigma(\varepsilon)$ as a normal graph (over an exhaustion of $\Sigma$ by compact subsets) for some functions $w(\varepsilon)$ and differentiating $w(\varepsilon)$ with respect to $\varepsilon$ at $\varepsilon=0$ gives rise to the desired Jacobi field.

Following [Kusner et al. 1996], we define the $6 k$-dimensional deficiency space

$$
\begin{aligned}
\mathscr{W}(\Sigma):=\bigoplus_{\ell=1}^{k} \operatorname{Span}\left\{\chi_{E_{\ell}} \Phi_{E_{\ell}}^{T, \vec{e}}:|\vec{e}|=\right. & 1\} \\
& \oplus \bigoplus_{\ell=1}^{k} \operatorname{Span}\left\{\chi_{E_{\ell}} \Phi_{E_{\ell}}^{D}\right\} \\
& \oplus \bigoplus_{\ell=1}^{k} \operatorname{Span}\left\{\chi_{E_{\ell}} \Phi_{E_{\ell}}^{R, \vec{e}^{\prime}}:\left|\vec{e}^{\prime}\right|=1, \vec{e}^{\prime} \cdot \vec{a}_{\ell}=0\right\},
\end{aligned}
$$

where $\chi_{E_{\ell}}$ is a cutoff function equal to 0 on $\Sigma-E_{\ell}$ and equal to 1 on $Y_{\ell}\left([1, \infty) \times S^{1}\right)$. The following proposition is the key result in the study of the structure of $\mathcal{M}_{g, k}^{\tau_{*}}$.

Proposition 3.5 ([Kusner et al. 1996]). Assume that $\Sigma \in \mathcal{M}_{g, k}^{\tau_{*}}$ is nondegenerate and fix $\mu \in\left(-\inf _{\ell} \gamma_{\tau_{\ell}, 2}, 0\right)$. Then the mapping

$$
\mathscr{L}_{\Sigma}: \mathscr{D}_{\mu}^{2, \alpha}(\Sigma) \oplus \mathscr{W}(\Sigma) \rightarrow \mathscr{D}_{\mu}^{0, \alpha}(\Sigma)
$$

is surjective and has a kernel of dimension $3 k$. In addition, there exists a $3 k$ dimensional subspace $\mathcal{N}(\Sigma) \subset \mathcal{W}(\Sigma)$ such that

$$
\operatorname{Ker} \mathscr{L}_{\Sigma} \subset \mathscr{D}_{\mu}^{2, \alpha}(\Sigma) \oplus \mathcal{N}(\Sigma) .
$$

Finally, given any $3 k$-dimensional subspace $\mathscr{K}(\Sigma) \subset \mathcal{W}(\Sigma)$ such that $\mathscr{K}(\Sigma) \oplus$ $\mathcal{N}(\Sigma)=\mathscr{W}(\Sigma)$, the mapping

$$
\mathscr{L}_{\Sigma}: \mathscr{D}_{\mu}^{2, \alpha}(\Sigma) \oplus \mathscr{K}(\Sigma) \rightarrow \mathscr{D}_{\mu}^{0, \alpha}(\Sigma)
$$

is an isomorphism.

It follows from this result that $M_{g, k}^{\tau_{*}}$ is locally a $3 k$-dimensional smooth manifold near any nondegenerate element [Kusner et al. 1996] (observe that we have not taken the quotient by the group of rigid motions of $\mathbb{R}^{3}$ ). 


\section{Building blocks}

We describe two families of complete noncompact constant mean curvature surfaces which will be used in the construction. The members of the first family are 3 -ended surfaces while the members of the second family are $k$-ended surfaces. We give a fairly precise description of the elements of each family and explain how these families can be obtained using already known constructions of complete noncompact constant mean curvature surfaces. In this paper we do not provide a proof of the existence of these families but rather to rely on their existence. We hope that the reader will either be convinced by the explanations below or take the existence of these families for granted.

Before we proceed with the description these surfaces, we recall the well known balancing formula [Korevaar et al. 1989]. Given a constant mean curvature surfaces $\Sigma \subset \mathbb{R}^{3}$ with finitely many ends $E_{\ell}$, for $\ell=1, \ldots, k$, which are asymptotic to Delaunay surfaces $D_{\tau_{\ell}}^{\vec{a}_{\ell}}+\vec{b}_{\ell}$, the balancing formula takes the form:

$$
\sum_{\ell=1}^{k} \tau_{\ell}\left|\tau_{\ell}\right| \vec{a}_{\ell}=0
$$

where $\vec{a}_{\ell}$ is the direction of the axis of $E_{\ell}$, which is normalized by $\left|\vec{a}_{\ell}\right|=1$ and points toward the end of $E_{\ell}$.

Type-1 surfaces. The members of the first family are denoted by $\Sigma_{\tau, \alpha}$, where $\tau$ and $\alpha$ are parameters. These surfaces are assumed to enjoy the following properties:

(i) Each $\Sigma_{\tau, \alpha}$ is a complete noncompact constant mean curvature surface with 3 ends which are denoted by $E_{-1}, E_{0}$ and $E_{1}$.

(ii) The surface $\Sigma_{\tau, \alpha}$ is invariant under the action of the group

$$
G:=\left\{I, S_{1}, S_{3}\right\}
$$

where $S_{i}$ is the symmetry with respect to the plane $x_{i}=0$.

(iii) Each $\Sigma_{\tau, \alpha}$ is nondegenerate and the parameters $(\tau, \alpha)$ are local parameters on the moduli space of constant mean curvature surfaces with 3 ends, which are invariant under the action of the group $G$.

(iv) The end $E_{0}$ is asymptotic to a Delaunay surface of parameter $\tau$ and axis the $x_{2}$-axis. The vector $-\vec{e}_{2}$ is directed toward the end of $E_{0}$. In particular, there exists a smooth function $(\tau, \alpha) \mapsto d_{0}(\tau, \alpha)$ such that $E_{0}$ is a graph (for an exponentially decaying function) over the Delaunay surface $\vec{D}_{\tau}^{\vec{e}_{2}}-d_{0} \vec{e}_{2}$. 
(v) The end $E_{1}$ is asymptotic to the Delaunay surface of parameter $\bar{\tau}$ and axis passing through the origin and of direction

$$
\vec{a}_{\alpha}:=\sin \alpha \vec{e}_{1}-\cos \alpha \vec{e}_{2} .
$$

The vector $\vec{a}_{\alpha}$ is directed toward the end of $E^{1}$. In particular, there exists a smooth function $(\tau, \alpha) \mapsto d_{1}(\tau, \alpha)$ such that $E_{1}$ is a graph (for an exponentially decaying function) over the Delaunay surface $D_{\bar{\tau}}^{\vec{a}_{\alpha}}+d_{1} \vec{a}_{\alpha}$.

(vi) The ends of $\Sigma_{\tau, \alpha}$ are regular.

Observe that the image of $E_{1}$ by $S_{1}$ is $E_{-1}$ and that $E_{0}$ remains globally fixed under the action of $S_{1}$. Also each end remains globally fixed under the action of $S_{3}$. Applying the balancing formula (4-1), we conclude that the Delaunay parameters $\bar{\tau}$ and $\tau$ are related by the formula

$$
\tau|\tau|+2 \cos \alpha \bar{\tau}|\bar{\tau}|=0 .
$$

In particular, if $\alpha \in(0, \pi / 2)$, the signs of $\tau$ and $\bar{\tau}$ are different and this implies that the surface $\Sigma_{\tau, \alpha}$ has always an end which is not embedded (asymptotic to a nodoid) in this case.

Observe that (iv) implies that the end $E_{0}$ can be parametrized by

$$
X_{\tau, \alpha}^{0}:=X_{\tau}^{\vec{e}_{2}}+w_{0} \vec{N}_{\tau}^{\vec{e}_{2}}-d_{0} \vec{e}_{2}
$$

with $(s, \theta) \in[0,+\infty) \times S^{1}$ and the function $w_{0} \in \mathscr{E}_{-\gamma_{\tau, 2}^{2, \alpha}}^{\mathscr{L}}\left([0,+\infty) \times S^{1}\right)$ depending on $\tau$ and $\alpha$. (In general the function $w_{0}$ is only defined on $[c,+\infty) \times S^{1}$ for some $c>0$ large enough. However, changing if necessary the value of $d_{0}(\tau, \alpha)$ by $2 m T_{\tau}$ for some $m \in \mathbb{N}$, we can assume that $w_{0}$ is defined on $[0,+\infty) \times S^{1}$.)

Similarly (v) implies that the end $E_{1}$ can be parametrized by

$$
X_{\tau, \alpha}^{1}:=X_{\bar{\tau}}^{\vec{a}_{\alpha}}+w_{1} \vec{N}_{\bar{\tau}}^{\vec{a}_{\alpha}}+d_{1} \vec{a}_{\alpha}
$$

with $(s, \theta) \in[0,+\infty) \times S^{1}$ and the function $w_{1} \in \mathscr{E}_{-\gamma_{\bar{\tau}, 2}^{2}}^{2, \alpha}\left([0,+\infty) \times S^{1}\right)$ depending on $\tau$ and $\alpha$. We denote by $X_{\tau, \alpha}^{-1}:=S_{1} \circ X_{\tau, \alpha}^{1}$, the parametrization of $E_{-1}$.

Definition 4.1. Given $s_{0}, s_{1}>0$, we define the compact surface with 3 boundaries

$$
\begin{aligned}
\Sigma_{\tau, \alpha}\left(s_{0}, s_{1}\right):=\Sigma_{\tau, \alpha}-\left(X_{\tau, \alpha}^{0}\left(\left(s_{0},+\infty\right) \times S^{1}\right) \cup X_{\tau, \alpha}^{1}\left(\left(s_{1},+\infty\right) \times S^{1}\right)\right. & \\
& \left.\cup X_{\tau, \alpha}^{-1}\left(\left(s_{1},+\infty\right) \times S^{1}\right)\right) .
\end{aligned}
$$

This is nothing but the surface $\Sigma_{\tau, \alpha}$ whose ends have been truncated.

Under the assumption of Alexandrov embeddedness, the surfaces described above have been classified in [Große-Brauckmann and Kusner 1999] and in [Korevaar et al. 2004] it is proved that they are nondegenerate. However, no such results are available in the case where the surfaces are not Alexandrov embedded, 
which is the case we have to consider! This is why we give now two examples of construction of such a family which rely on connected sum constructions and for which it is possible to check that the surfaces constructed are both nondegenerate and have regular ends:

Example 4.2. A first family can be obtained by gluing onto the unit sphere $S^{2} \subset \mathbb{R}^{3}$, three half Delaunay surfaces of parameters $\bar{\tau}, \tau$ and $\bar{\tau}$, respectively, at the points having coordinates

$$
(-\sin \alpha,-\cos \alpha, 0), \quad(0,-1,0) \quad \text { and } \quad(\sin \alpha,-\cos \alpha, 0),
$$

using a modified version of the connected sum result of [Mazzeo et al. 2001; 2005] (see also [Jleli 2004]). The construction works if one demands that the surfaces be invariant under the action of the group $G$. Given the symmetries of the surfaces constructed, there remain only two degrees of freedom: the Delaunay parameter $\tau$ and the angle $\alpha$ between the ends. The construction works for any $\alpha \in(0, \pi / 2) \cup$ $(\pi / 2, \pi)$ and any $\tau \neq 0$ close enough to 0 . The fact that the ends are regular follows from the construction itself since $\tau$ can be used to parameterize this family of surfaces and differentiation with respect to this parameter yields a Jacobi field whose asymptotic along any end has a nontrivial component on $\chi_{E_{\ell}} \Phi_{E_{\ell}}^{D}$, for $\ell=$ $0, \pm 1$.

Example 4.3. A second family can be obtained by gluing onto a Delaunay surface of parameter $\bar{\tau}$ and axis $x_{1}$ which is translated so that it is invariant under the action of the symmetry $S_{1}$ (namely either $D_{\bar{\tau}}^{\vec{e}_{1}}$ or $D_{\bar{\tau}}^{\vec{e}_{1}}+T_{\bar{\tau}} \vec{e}_{1}$ ), a half Delaunay surface of axis $x_{2}$ and small Delaunay parameter $\tau$. Again, the construction works if one demands that the surfaces be invariant under the action of the group $G$. Given the symmetries of the surfaces constructed, there remains only two degrees of freedom: the Delaunay parameters $\bar{\tau}$ and $\tau$. The construction works for any small value of the parameter $\tau \neq 0$ ([Mazzeo et al. 2001; 2005]; see also [Jleli 2004]) and provides a surface with an angle $\alpha$ close, but not equal, to $\pi / 2$ which is determined by the equation $\tau|\tau|+2 \cos \alpha \bar{\tau}|\bar{\tau}|=0$. This shows that $(\alpha, \tau)$ are local parameters on the corresponding moduli space and, as in the previous example, the ends of the surfaces are regular.

In both cases, the surfaces can be seen to be nondegenerate, when $\tau$ is close enough to 0, using the strategy developed in [Mazzeo and Pacard 2001].

Type-2 surfaces. We fix $k \geq 3$. The members of the second family are denoted by $\bar{\Sigma}_{\tau}$, where $\tau$ is a parameter. These surfaces are assumed to enjoy the following properties:

(i) Each $\bar{\Sigma}_{\tau}$ is a complete noncompact constant mean curvature surface with $k$ ends which are denoted by $\bar{E}_{0}, \ldots, \bar{E}_{k-1}$. 
(ii) The surface is invariant under the action of the group

$$
G_{k}:=\left\{R_{2 \pi j / k}: j \in \mathbb{Z}\right\}
$$

where $R_{\theta}$ is the rotation of angle $\theta$ in the $x_{1}, x_{2}$ plane.

(iii) Each $\bar{\Sigma}_{\tau}$ is nondegenerate and the parameter $\tau$ is a local parameter on the moduli space of constant mean curvature surfaces with $k$ ends, which are invariant under the action of the group $G_{k}$.

(iv) The end $\bar{E}_{0}$ is asymptotic to a Delaunay surface having parameter $\tau$ and $x_{2}$ axis as its axis, the vector $\vec{e}_{2}$ being directed toward the end of $\bar{E}_{0}$. In particular, there exists a smooth function $\tau \mapsto \bar{d}_{0}(\tau)$ such that $\bar{E}_{0}$ is a graph (for an exponentially decaying function) over the Delaunay surface $D_{\tau}^{\vec{e}_{2}}+\vec{d}^{0} \vec{e}_{2}$.

(v) The ends of $\bar{\Sigma}_{\tau}$ are regular.

For $\ell=1, \ldots, k-1$, the image of $E_{0}$ by $R_{2 \pi \ell / k}$ is the end $\bar{E}_{\ell}$. Hence the angle between two consecutive ends is given by $2 \pi / k$, and to check that the ends of $\bar{\Sigma}_{\tau}$ are regular it is enough to check that $\bar{E}_{0}$ is regular.

As in the case of Type- 1 surfaces, statement (iv) implies that the end $\bar{E}_{\tau}^{0}$ can be parametrized by

$$
\bar{X}_{\tau}^{0}:=X_{\tau}^{\vec{e}_{2}}+\bar{w}_{0} \vec{N}_{\tau}^{\vec{e}_{2}}+\bar{d}_{0} \vec{e}_{2}
$$

with $(s, \theta) \in[0,+\infty) \times S^{1}$, for some function $\bar{w}_{0} \in \mathscr{E}_{-\gamma_{\tau, 2}}^{2, \alpha}\left([0,+\infty) \times S^{1}\right)$ which depends on $\tau$. We denote by $\bar{X}_{\tau}^{\ell}:=R_{2 \pi \ell / k} \circ \bar{X}_{\tau}^{0}$, the parametrization of $\bar{E}_{\ell}$.

Definition 4.4. Given $s_{0}>0$, we define the compact surface with $k$ boundaries

$$
\bar{\Sigma}_{\tau}\left(s_{0}\right):=\bar{\Sigma}_{\tau}-\bigcup_{\ell=0}^{k-1} \bar{X}_{\tau}^{\ell}\left(\left(s_{0},+\infty\right) \times S^{1}\right) .
$$

This is nothing but $\bar{\Sigma}_{\tau}$ with all the ends truncated. We now give two examples of such a family.

Example 4.5. A first family can be obtained by gluing onto the unit sphere $S^{2} \subset \mathbb{R}^{3}$, $k$ copies of a half Delaunay surface with small Delaunay parameter $\tau \neq 0$ in such a way that the surface remains invariant under the action of $G_{k}$. Again, this is a byproduct of the end addition result proved in [Mazzeo et al. 2001; 2005] or this is also a byproduct of [Kapouleas 1990]. These surfaces have also been constructed and described by K. Große-Brauckmann [1993].

Example 4.6. A second family can be obtained by gluing onto a $k$-noid (a minimal surface with $k$ ends of catenoidal type [Jorge and Meeks 1983; Cosín and Ros 2001]) which is invariant under the action of $G_{k}, k$ copies of a half Delaunay surface with small Delaunay parameter $\tau \neq 0$ in such a way that the symmetries are preserved. This construction is the one described in [Mazzeo and Pacard 2001]. 
In either case, given the symmetries of the surfaces constructed, there remains only one degree of freedom which is $\tau$, the Delaunay parameter of the ends. Both constructions work for any $\tau \neq 0$ close enough to 0 . The fact that (v) holds follows at once from the construction itself since $\tau$ can be used to parameterize this family of surfaces and differentiation with respect to this parameter yields a Jacobi field whose asymptotic has a nontrivial component on $\chi_{\bar{E}_{0}} \Phi_{\bar{E}_{0}}^{D}$. The fact that the surfaces constructed are nondegenerate follows from [Mazzeo and Pacard 2001].

Jacobi fields. We give a precise description of the Jacobi fields on both $\Sigma_{\tau, \alpha}$ and on $\bar{\Sigma}_{\tau}$. This description induces a description of the spaces $\mathscr{K}\left(\Sigma_{\tau, \alpha}\right)$ and $\mathscr{K}\left(\bar{\Sigma}_{\tau}\right)$ which have been introduced in Proposition 3.5.

Jacobi fields on $\bar{\Sigma}_{\tau}$. We start with the analysis of the Jacobi fields on $\bar{\Sigma}_{\tau}$ since this is the simplest. Since the surface $\bar{\Sigma}_{\tau}$ is assumed to be nondegenerate, the deficiency space $\mathcal{W}\left(\bar{\Sigma}_{\tau}\right)$ is $6 k$-dimensional. However, since we are working in the space of surfaces which are invariant under the action of the group $G_{k}$, this reduces the dimension of the corresponding moduli space to 1 and the deficiency space is now spanned by the 2 functions

$$
\bar{\Psi}^{T}:=\sum_{\ell=0}^{k-1} \chi_{\bar{E}_{\ell}} \Phi_{\bar{E}_{\ell}}^{T, \vec{a}_{\ell}} \quad \text { and } \quad \bar{\Psi}^{D}:=\sum_{\ell=0}^{k-1} \chi_{\bar{E}_{\ell}} \Phi_{\bar{E}_{\ell}}^{D}
$$

where $\vec{a}_{\ell}:=R_{2 \pi \ell / k} \vec{e}_{2}$ is the direction of the end $\vec{E}_{\ell}$. Even though these functions do depend on $\tau$, we have not made this dependence apparent in the notation. Observe that the symmetries of $\bar{\Sigma}_{\tau}$ imply that

$$
\Phi_{\bar{E}_{\ell}}^{T, \vec{a}_{\ell}}=\Phi_{\bar{E}_{0}}^{T, \vec{e}_{2}} \circ\left(R_{2 \pi \ell / k}\right)^{-1} .
$$

Since the end $\bar{E}_{0}$ is assumed to be regular, there exists a globally defined Jacobi field $\bar{\Phi}^{D}$ (invariant under the action of $G_{k}$ ) whose asymptotic on $\bar{E}_{0}$ has a nontrivial component on $\Phi_{\bar{E}_{0}}^{D}$. In fact this Jacobi field is obtained by moving the parameter $\tau$. Multiplying this Jacobi field by a suitable constant, we can assume that it is asymptotic to $\bar{\Psi}^{D}+\bar{c} \bar{\Psi}^{T}$ on each $\bar{E}_{\ell}$, were the constant $\bar{c}$ depends smoothly upon $\tau$. This implies that the space $\mathscr{K}\left(\bar{\Sigma}_{\tau}\right)$ can be chosen to be

$$
\mathscr{K}\left(\bar{\Sigma}_{\tau}\right)=\operatorname{Span}\left\{\bar{\Psi}^{T}\right\}
$$

Jacobi fields on $\Sigma_{\tau, \alpha}$. We now analyze the Jacobi fields on $\Sigma_{\tau, \alpha}$. By assumption, $\Sigma_{\tau, \alpha}$ is nondegenerate and has 3 ends, therefore the deficiency space $\mathscr{W}\left(\Sigma_{\tau, \alpha}\right)$ is 18-dimensional. Now, recall that we are working in the space of surfaces which are invariant under the action of the group $G$ and this reduces the dimension of the corresponding moduli space to 3 and the deficiency space is spanned by the 6 
functions we now describe:

$$
\begin{array}{rlrl}
\Psi^{T} & :=\chi_{E_{0}} \Phi_{E_{0}}^{T, \vec{e}_{2}}, & \Psi^{D} & :=\chi_{E_{0}} \Phi_{E_{0}}^{D}, \\
\Psi^{\bar{D}} & :=\chi_{E_{1}} \Phi_{E_{1}}^{D}+\left(\chi_{E_{1}} \Phi_{E_{1}}^{D}\right) \circ S_{1}, & \Psi^{T, \vec{a}}:=\chi_{E_{1}} \Phi_{E_{1}}^{T, \vec{a}_{\alpha}}+\left(\chi_{E_{1}} \Phi_{E_{1}}^{T, \vec{a}_{\alpha}}\right) \circ S_{1}, \\
\Psi^{T, \vec{a}^{\perp}}: & :=\chi_{E_{1}} \Phi_{E_{1}}^{T, \vec{a}_{\alpha}^{\perp}}+\left(\chi_{E_{1}} \Phi_{E_{1}}^{T, \vec{a}_{\alpha}^{\perp}}\right) \circ S_{1}, & \Psi^{R}:=\chi_{E_{1}} \Phi_{E_{1}}^{R, \vec{a}_{\alpha}^{\perp}}+\left(\chi_{E_{1}} \Phi_{E_{1}}^{R, \vec{a}_{\alpha}^{\perp}}\right) \circ S_{1},
\end{array}
$$

where

$$
\vec{a}:=\vec{a}_{\alpha} \quad \text { and } \quad \vec{a}^{\perp}:=\vec{a}_{\alpha}^{\perp}:=\cos \alpha \vec{e}_{1}+\sin \alpha \vec{e}_{2} .
$$

Even though these functions clearly depend on $\alpha$ and $\tau$, we have not made this dependence apparent in the notation. Observe that $\Psi^{D}$ and $\Psi^{T}$ are supported on $E_{0}$ while $\Psi^{\bar{D}}, \Psi^{T, \vec{a}}, \Psi^{T, \vec{a}^{\perp}}$ and $\Psi^{R}$ are supported on $E_{1} \cup E_{-1}$.

We now describe the Jacobi fields which are globally defined on $\Sigma_{\tau, \alpha}$ and which are obtained by moving the two parameters $\alpha, \tau$ and also by translating this surface in the $\vec{e}_{2}$ direction. These Jacobi fields will give us an explicit description of $\mathcal{N}\left(\Sigma_{\tau, \alpha}\right)$ and hence a description of some choice of $\mathscr{K}\left(\Sigma_{\tau, \alpha}\right)$.

(1) Changing the parameter $\tau$ (keeping $\alpha$ fixed) changes $\bar{\tau}$ and provides a Jacobi field which (up to a multiplicative constant) is asymptotic to $\Psi^{\bar{D}}+c_{1} \Psi^{T, \vec{a}}$ on $E_{1} \cup E_{-1}$ and which is asymptotic to $c_{2} \Psi^{D}+c_{3} \Psi^{T}$ on $E_{0}$.

(2) Changing the $\alpha$ parameter (keeping $\tau$ fixed) provides a Jacobi field which (up to a multiplicative constant) is asymptotic to $\Psi^{R}+c_{4} \Psi^{T, \vec{a}}+c_{5} \Psi^{D}+c_{6} \Psi^{T, \vec{a}^{\perp}}$ on $E_{1} \cup E_{-1}$ and which is asymptotic to $c_{7} \Psi^{T}$ on $E_{0}$.

(3) Translating $\Sigma_{\tau, \alpha}$ in the $\vec{e}_{2}$ direction (keeping $\tau$ and $\alpha$ fixed) provides a Jacobi field

$$
\Phi^{T, \vec{e}_{2}}:=\vec{N}_{\Sigma_{\tau, \alpha}} \cdot \vec{e}_{2}
$$

which is asymptotic to $\Psi^{T}$ on $E_{0}$ and which is asymptotic to $c_{8} \Psi^{T, \vec{a}}+c_{9} \Psi^{T, \vec{a}^{\perp}}$ on $E_{1} \cup E_{-1}$.

Here the constants $c_{1}, \ldots, c_{9}$ are smooth functions of $\tau$ and $\alpha$. Recall that the space $\mathscr{K}\left(\Sigma_{\tau, \alpha}\right)$ is any 3 -dimensional subspace of the deficiency space $\mathscr{W}\left(\Sigma_{\tau, \alpha}\right)$ chosen so that

$$
\mathscr{W}\left(\Sigma_{\tau, \alpha}\right)=\mathscr{K}\left(\Sigma_{\tau, \alpha}\right) \oplus \mathcal{N}\left(\Sigma_{\tau, \alpha}\right) .
$$

It follows from the above description of the elements of $\mathcal{N}\left(\Sigma_{\tau, \alpha}\right)$ that we can choose

$$
\mathscr{K}\left(\Sigma_{\tau, \alpha}\right)=\operatorname{Span}\left\{\Psi^{D}, \Psi^{T, \vec{a}}, \Psi^{T, \vec{a}^{\perp}}\right\} .
$$

For all $t \in \mathbb{R}$, we define

$$
\mathscr{K}\left(\Sigma_{\tau, \alpha}, t\right)=\operatorname{Span}\left\{\Psi^{D}+t \Phi^{T, \vec{e}_{2}}, \Psi^{T, \vec{a}}, \Psi^{T, \vec{a}^{\perp}}\right\} .
$$


Observe that, $\Phi^{T, \vec{e}_{2}}$ being a globally defined Jacobi field, the space $\mathscr{K}\left(\Sigma_{\tau, \alpha}\right)$ can be replaced by $\mathscr{T}\left(\Sigma_{\tau, \alpha}, t\right)$ in the statement of Proposition 3.5 corresponding to $\Sigma_{\tau, \alpha}$.

\section{The construction}

We fix $k \geq 3$ and define

$$
\alpha_{k}:=\frac{\pi}{2}-\frac{\pi}{k}
$$

We assume that, for $\tau$ in some closed interval $I \subset\left(\tau_{*}, 0\right) \cup(0,1)$ with nonempty interior, we are given a family of surfaces $\Sigma_{\tau, \alpha_{k}}$ of Type 1 and a family of surfaces $\bar{\Sigma}_{\tau}$ of Type 2. The parameter $\tau$ being chosen in $I$, we recall that $\bar{\tau}$ is given by

$$
\tau|\tau|+2 \cos \alpha_{k} \bar{\tau}|\bar{\tau}|=0 .
$$

Given $n, m \in \mathbb{N}$, we set

$$
\delta_{n, \tau}:=d_{0}\left(\tau, \alpha_{k}\right)+\bar{d}_{0}(\tau)+2 n T_{\tau} .
$$

We agree on the notation

$$
\Sigma_{n, \tau}:=\Sigma_{\tau, \alpha_{k}}+\delta_{n, \tau} \vec{e}_{2}
$$

and denote the ends of this surface by

$$
E_{\ell}^{n}:=E_{\ell}+\delta_{n, \tau} \vec{e}_{2} .
$$

Also we define the truncated surface (see Definition 4.1)

$$
\Sigma_{n, \tau}\left(s_{0}, s_{1}\right):=\Sigma_{\tau, \alpha_{k}}\left(s_{0}, s_{1}\right)+\delta_{n, \tau} \vec{e}_{2} .
$$

With these notations in mind, we consider the union of the surface $\Sigma_{n, \tau}\left(n s_{\tau}, m s_{\bar{\tau}}\right)$ with the images of this surface by $R_{2 \ell \pi / k}$, for $\ell=1, \ldots, k-1$ and also the truncated surface $\bar{\Sigma}_{\tau}\left(n s_{\tau}\right)$ (see Definition 4.4). These surfaces with boundaries are now connected together using appropriate cutoff functions to produce a compact surface which is invariant under the action of $G_{k}$. More precisely, for each $\ell=0, \ldots, k-1$, the end $\bar{E}_{\ell}$ of $\bar{\Sigma}_{\tau}$ can be connected with the image of $E_{0}^{n}$ by $R_{2 \pi \ell / k}$ since they are graphs over the same Delaunay surface. And, provided the parameters $n, m$ and $\tau$ are suitably chosen, the image of the end $E_{1}^{n}$ by $R_{2 \pi \ell / k}$ can be connected with the image of $E_{-1}^{n}$ by $R_{2 \pi(\ell+1) / k}$. We now describe this procedure analytically. Given that the surface we want to construct should be invariant under the action of $G_{k}$, here is all we need to describe:

Connection of $E_{0}^{n}$ with $\bar{E}_{0}$. By construction the ends $E_{0}^{n}$ and $\bar{E}_{0}$ are normal graphs over the same Delaunay surface. Given the parametrizations defined in (4-3) and (4-5) we can connect the two pieces together by considering the parametrization

$$
Y_{n, \tau}^{0}(s, \theta):=\xi(s) \bar{X}_{\tau}^{0}\left(s+n s_{\tau}, \theta\right)+(1-\xi(s))\left(X_{\tau, \alpha_{k}}^{0}\left(n s_{\tau}-s, \theta\right)+\delta_{n, \tau} \vec{e}_{2}\right)
$$


for $(s, \theta) \in\left(-n s_{\tau}, n s_{\tau}\right) \times S^{1}$. Here $\xi$ is a cutoff function identically equal to 1 for $s \leq-1$ and identically equal to 0 for $s \geq 1$ and which satisfies

$$
\xi(-s)=1-\xi(s) .
$$

We will denote by $A_{n, \tau}^{0}$ the image of $(-1,1) \times S^{1}$ by $Y_{n, \tau}^{0}$.

We define, for $\ell=1, \ldots, k-1$

$$
Y_{n, \tau}^{\ell}=R_{2 \pi \ell / k} \circ Y_{n, \tau}^{0}
$$

to be the parametrization which describes the connected sum between the end $\bar{E}_{\ell}$ and the image of $E_{0}^{n}$ by $R_{2 \pi \ell / k}$.

Connection of $E_{1}^{n}$ with the image of $E_{-1}^{n}$ by $R_{2 \pi / k}$. We define the plane

$$
\Pi_{k}:=\left\{x \in \mathbb{R}^{3}: \tan (2 \pi / k) x_{2}=-x_{1}\right\} .
$$

The image of $E_{1}^{n}$ by the symmetry with respect to $\Pi_{k}$ is equal to the image of $E_{-1}^{n}$ by $R_{2 \pi / k}$. By definition, the end $E_{1}^{n}$ is a graph over the Delaunay surface $D_{\bar{\tau}}^{\vec{a}}+d^{1} \vec{a}+\delta_{n, \tau} \vec{e}_{2}$. Therefore the end $E_{1}^{n}$ and its image by the symmetry with respect to the plane $\Pi_{k}$ are normal graphs over the same Delaunay surface if and only if the Delaunay surface $D_{\bar{\tau}}^{\vec{a}}+d^{1} \vec{a}+\delta_{n, \tau} \vec{e}_{2}$ is invariant under the symmetry with respect to the plane $\Pi_{k}$. This condition is translated into the fact that the integer $m \in \mathbb{N}$ is chosen so that

$$
\sin (\pi / k)\left(d^{0}\left(\tau, \alpha_{k}\right)+\bar{d}^{0}(\tau)+2 n T_{\tau}\right)=d^{1}\left(\tau, \alpha_{k}\right)+m T_{\bar{\tau}}
$$

where we recall that the parameters $\bar{\tau}$ and $\tau$ are related by

$$
\tau|\tau|+2 \sin (\pi / k) \bar{\tau}|\bar{\tau}|=0 .
$$

If (5-2) is fulfilled we can connect the end $E_{1}^{n}$ with the image of the end $E_{-1}^{n}$ by $R_{2 \pi / k}$, using the parametrization

$Z_{n, \tau}^{0}(s, \theta):=\xi(s) X_{\tau, \alpha_{k}}^{1}\left(s+m s_{\bar{\tau}}, \theta\right)+(1-\xi(s)) R_{2 \pi / k} \circ X_{\tau, \alpha_{k}}^{-1}\left(m s_{\bar{\tau}}-s, \theta\right)+\delta_{n, \tau} \vec{e}_{2}$ for $(s, \theta) \in\left(-m s_{\bar{\tau}}, m s_{\bar{\tau}}\right) \times S^{1}$. We will denote by $A_{n, \tau}^{1}$ the image of $(-1,1) \times S^{1}$ by $Z_{n, \tau}^{0}$.

We define

$$
Z_{n, \tau}^{\ell}:=R_{2 \pi \ell / k} \circ Z_{n, \tau}^{0}
$$

for $\ell=1, \ldots, k-1$. This describes the connection of the image of $E_{1}^{n}$ by $R_{2 \pi \ell / k}$ with the image of $E_{-1}^{n}$ by $R_{2 \pi(\ell+1) / k}$. 
Estimate of the mean curvature of the connected surface. The compact surface which is obtained through these connections will be denoted by $S_{n, \tau}$. It is an immersed compact surface of genus $k$. By construction, the mean curvature of the surface $S_{n, \tau}$ is equal to 1 except in annular regions $A_{n, \tau}^{0}, A_{n, \tau}^{1}$ and in their images by the elements of $G_{k}$. The following estimates follow at once from the exponential decay of the functions $w_{0}, \bar{w}_{0}$ and $w_{1}$ (see Section 4 ).

Lemma 5.1. We have

$$
\left\|H_{S_{n, \tau}}-1\right\|_{\mathscr{C}^{0, \alpha}\left(A_{n, \tau}^{0}\right)} \leq c e^{-n \gamma_{\tau, 2} S_{\tau}}
$$

and, provided (5-2) is satisfied, we have

$$
\left\|H_{S_{n, \tau}}-1\right\|_{\mathscr{C}^{0, \alpha}\left(A_{n, \tau}^{1}\right)} \leq c e^{-m \gamma_{\bar{\tau}, 2} s_{\bar{\tau}}}
$$

where the constant $c>0$ does not depend on $\tau \in I$ nor on $n \in \mathbb{N}$.

Partition of unity on $S_{n, \tau}$. We now describe a partition of unity subordinate to the construction above.

(i) The function $\chi_{n, \tau}$ is a smooth function defined on $S_{n, \tau}$, equal to 1 on

$$
\Sigma_{n, \tau}\left(n s_{\tau}-1, m s_{\bar{\tau}}-1\right) \subset S_{n, \tau}
$$

and equal to 0 on the complement of

$$
\begin{aligned}
\Sigma_{n, \tau}\left(n s_{\tau}-1, m s_{\bar{\tau}}-1\right) \cup Y_{n, \tau}^{0}\left((-1,1) \times S^{1}\right) \cup Z_{n, \tau}^{0}\left((-1,1) \times S^{1}\right) & \\
\cup & Z_{n, \tau}^{k-1}\left((-1,1) \times S^{1}\right)
\end{aligned}
$$

in $S_{n, \tau}$. To be more precise, on the part of $S_{n, \tau}$ parametrized by $Y_{n, \tau}^{0}$, the function $\chi_{n, \tau}$ is equal to 1 for $s \geq 1$ and is equal to 0 for $s \leq-1$ and on the part of $S_{n, \tau}$ parametrized by $Z_{n, \tau}^{0}$, the function $\chi_{n, \tau}$ is equal to 1 for $s \leq-1$ and equal to 0 for $s \geq 1$. This function is assumed to be invariant under the action of $S_{1}$.

(ii) The function $\bar{\chi}_{n, \tau}$ is a smooth function on $S_{n, \tau}$, defined by

$$
\bar{\chi}_{n, \tau}=1-\sum_{\ell=0}^{k-1} \chi_{n, \tau} \circ R_{2 \pi \ell / k} .
$$

This function is equal to 1 on

$$
\bar{\Sigma}_{\tau}\left(n s_{\tau}-1\right) \subset S_{n, \tau}
$$

and equal to 0 on the complement of

$$
\bar{\Sigma}_{\tau}\left(n s_{\tau}-1\right) \cup \bigcup_{\ell=0}^{k-1} Y_{n, \tau}^{\ell}\left((-1,1) \times S^{1}\right)
$$


in $S_{n, \tau}$. To be more precise, on the part of $S_{n, \tau}$ parametrized by $Y_{n, \tau}^{0}$, the function $\bar{\chi}_{n, \tau}$ is equal to 1 for $s \leq-1$ and equal to 0 for $s \geq 1$. This function is assumed to be invariant under the action of $G_{k}$.

We will also need another set of cutoff functions, as follows:

(i) The function $\chi_{n, \tau}^{e}$ is a smooth function defined on $S_{n, \tau}$, equal to 1 on

$$
\begin{aligned}
\Sigma_{n, \tau}\left(n s_{\tau}-1, m s_{\bar{\tau}}-1\right) \cup Y_{n, \tau}^{0}\left(\left(-n s_{\tau}+2,1\right) \times S^{1}\right) \\
\cup Z_{n, \tau}^{0}\left(\left(-1, m s_{\bar{\tau}}-2\right) \times S^{1}\right) \cup Z_{n, \tau}^{k-1}\left(\left(-1, m s_{\bar{\tau}}-2\right) \times S^{1}\right)
\end{aligned}
$$

and equal to 0 on the complement of

$$
\begin{aligned}
\Sigma_{n, \tau}\left(n s_{\tau}-1, m s_{\bar{\tau}}-1\right) \cup Y_{n, \tau}^{0}\left(\left(-n s_{\tau}+1,1\right) \times S^{1}\right) \\
\cup Z_{n, \tau}^{0}\left(\left(-1, m s_{\bar{\tau}}-1\right) \times S^{1}\right) \cup Z_{n, \tau}^{k-1}\left(\left(-1, m s_{\bar{\tau}}-1\right) \times S^{1}\right) .
\end{aligned}
$$

To be more precise, on the part of $S_{n, \tau}$ parametrized by $Y_{n, \tau}^{0}$, the function $\chi_{n, \tau}^{e}$ is equal to 1 for $s \geq-n s_{\tau}+2$ and equal to 0 for $s \leq-n s_{\tau}+1$ and on the part of $S_{n, \tau}$ parametrized by $Z_{n, \tau}^{0}$, the function $\chi_{n, \tau}^{e}$ is equal to 1 for $s \leq m s_{\bar{\tau}}-2$ and equal to 0 for $s \geq m s_{\bar{\tau}}-1$. This function is assumed to be invariant under the action of $S_{1}$.

(ii) The function $\bar{\chi}_{n, \tau}^{e}$ is a smooth function defined on $S_{n, \tau}$, equal to 1 on

$$
\bar{\Sigma}_{\tau}\left(n s_{\tau}-1\right) \cup \bigcup_{\ell=0}^{k-1} Y_{n, \tau}^{\ell}\left(\left(0, n s_{\tau}-2\right) \times S^{1}\right)
$$

and equal to 0 on the complement of

$$
\bar{\Sigma}_{\tau}\left(n s_{\tau}-1\right) \cup \bigcup_{\ell=0}^{k-1} Y_{n, \tau}^{\ell}\left(\left(0, n s_{\tau}-1\right) \times S^{1}\right) .
$$

To be precise, on the part of $S_{n, \tau}$ parametrized by $Y_{n, \tau}^{0}$, the function $\bar{\chi}_{n, \tau}^{e}$ is equal to 1 for $s \leq n s_{\tau}-2$ and equal to 0 for $s \geq n s_{\tau}-1$. This function is assumed to be invariant under the action of $G_{k}$.

\section{Linear analysis}

Extension of the elements of $\mathscr{K}\left(\Sigma_{n, \tau}, t\right)$ and $\mathscr{K}\left(\bar{\Sigma}_{\tau}\right)$. Building on our analysis of Jacobi fields (page 95 and following), we explain how the restriction of the elements of $\mathscr{K}\left(\Sigma_{n, \tau}, t\right)$ to $\Sigma_{n, \tau}\left(n s_{\tau}-1, m s_{\tau}-1\right) \subset S_{n, \tau}$ and the restriction of the elements of $\mathscr{K}\left(\bar{\Sigma}_{\tau}\right)$ to $\bar{\Sigma}_{\tau}\left(n s_{\tau}-1\right) \subset S_{n, \tau}$ can be extended to functions which are defined on $S_{n, \tau}$, using the restrictions of the elements of the nullspace of $\mathscr{L}_{\Sigma_{n, \tau}}$ to $\Sigma_{n, \tau}\left(n s_{\tau}-1, m s_{\tau}-1\right)$ and the restriction of the elements of the nullspace of $\mathscr{L}_{\bar{\Sigma}_{\tau}}$ to $\bar{\Sigma}_{\tau}\left(n s_{\tau}-1\right)$ and also using the action of $G_{k}$. The fact that these extensions are 
meaningful (see Lemma 6.1) relies on the fact that we have chosen $m$ satisfying (5-2).

Warning. We will keep using the same notations for the elements of $\mathscr{K}\left(\Sigma_{n, \tau}, t\right)$ and $\mathscr{K}\left(\Sigma_{\tau, \alpha_{k}}, t\right)$.

We now reason as follows.

(i) The restriction of the elements $\Psi^{T, \vec{a}}, \Psi^{T, \vec{a}^{\perp}} \in \mathscr{K}\left(\Sigma_{n, \tau}, t\right)$ to $\Sigma_{n, \tau}\left(n s_{\tau}, m s_{\bar{\tau}}\right)$ can be easily extended to $S_{n, \tau}$ using the fact that the ends $E_{1}^{n}$ and $R_{2 \pi / k} E_{-1}^{n}$ are symmetric with respect to $\Pi_{k}$. For example, for $\vec{b}=\vec{a}, \vec{a}^{\perp}$, we can first define a function $\Psi_{n}^{T, \vec{b}}$ on the part of $S_{n, \tau}$ which is parametrized by $Z_{n, \tau}^{1}$,

$$
\Psi_{n}^{T, \vec{b}}=\chi_{n, \tau} \Psi^{T, \vec{b}}+\left(1-\chi_{n, \tau}\right) \Psi^{T, \vec{b}} \circ\left(R_{2 \pi / k}\right)^{-1}
$$

and then use the action of $G_{k}$ to extend this function to the other components of $S_{n, \tau}$.

(ii) The restriction of the element $\bar{\Psi}^{T} \in \mathscr{T}\left(\bar{\Sigma}_{\tau}\right)$ to $\bar{\Sigma}_{\tau}\left(n s_{\tau}-1\right)$ can be extended to $S_{n, \tau}$ using the restriction to $\Sigma_{n, \tau}\left(n, s_{\tau}-1, m, s_{\bar{\tau}}-1\right)$ of $\Phi^{T, \vec{e}_{2}}$, the (unique) Jacobi field defined on $\Sigma_{n, \tau}$ which is asymptotic to $\Phi_{E_{0}^{n}}^{T, \vec{e}_{2}}$ on $E_{0}^{n}$ (i.e. the globally defined Jacobi field which corresponds to translation of $\Sigma_{n, \tau}$ along the $x_{2}$ axis). We define a function $\bar{\Psi}_{n}^{T}$ first by writing

$$
\bar{\Psi}_{n}^{T}=\bar{\chi}_{n, \tau} \bar{\Psi}^{T}+\left(1-\bar{\chi}_{n, \tau}\right) \Phi^{T, \vec{e}_{2}}
$$

on the part of $S_{n, \tau}$ which is parametrized by $Y_{n, \tau}^{0}$. Observe that $\Phi^{T, \vec{e}_{2}}$ is asymptotic to a linear combination of $\Psi^{T, \vec{a}}$ and $\Psi^{T, \vec{a}^{\perp}}$ on the other ends of $\Sigma_{n, \tau}$ and we can use the type of extension described in (i) to extend the function to $S_{n, \tau}$. For example,

$$
\bar{\Psi}_{n}^{T}=\chi_{n, \tau} \Phi^{T, \vec{e}_{2}}+\left(1-\chi_{n, \tau}\right) \Phi^{T, \vec{e}_{2}} \circ\left(R_{2 \pi / k}\right)^{-1}
$$

on the part of $S_{n, \tau}$ which is parametrized by $Z_{n, \tau}^{0}$, and then we use the action of $G_{k}$ to extend this function to the other components of $S_{n, \tau}$.

(iii) Finally, it remains to explain how to choose the parameter $t$ (defined in the construction of $\left.\mathscr{K}\left(\Sigma_{n, \tau}, t\right)\right)$ in such a way that the element

$$
\Psi^{D}+t \Phi^{T, \vec{e}_{2}} \in \mathscr{K}\left(\Sigma_{n, \tau}, t\right)
$$

is asymptotic to $\bar{\Phi}^{D}$, the (unique) Jacobi field defined on $\bar{\Sigma}_{\tau}$ which is asymptotic to $\Phi_{\bar{E}_{0}}^{D}$ on $\bar{E}_{0}$ (i.e. the globally defined Jacobi field which corresponds to changes of Delaunay parameters of $\bar{\Sigma}_{\tau}$ ). To be more specific, $\left(\Psi^{D}+t \Phi^{T, \vec{e}_{2}}\right) \circ Y_{n, \tau}^{0}$ is asymptotic to

$$
\Phi_{D_{\tau}}^{D}\left(\cdot-n s_{\tau}, \cdot\right)+t \Phi_{D_{\tau}}^{T, \vec{e}_{2}}\left(\cdot-n s_{\tau}, \cdot\right)
$$


and $\Phi_{\bar{E}_{\tau}^{0}}^{D} \circ \bar{Y}_{n, \tau}^{0}$ is asymptotic to

$$
\Phi_{D_{\tau}}^{D}\left(\cdot+n s_{\tau}, \cdot\right) .
$$

Granted (2-2), it is enough to choose

$$
t=2 n \partial_{\tau} T_{\tau}
$$

These two functions are then connected, as in (i) or (ii), to define the function $\Psi_{n}^{D}$. For example, we define

$$
\Psi_{n}^{D}=\bar{\chi}_{n, \tau} \Phi_{\bar{E}_{\tau}^{0}}^{D}+\left(1-\bar{\chi}_{n, \tau}\right)\left(\psi_{E^{0}}^{D}+n \partial_{\tau} T_{\tau} \Phi_{\Sigma_{n, \tau}}^{T, \vec{e}_{2}}\right)
$$

on the part of $S_{n, \tau}$ which is parametrized by $Y_{n, \tau}^{0}$ and then we extend this function to all $S_{n, \tau}$ using the action of $G_{k}$.

We define $\mathscr{L}_{S_{n, \tau}}$ to be the Jacobi operator about the surface $S_{n, \tau}$. The following result again follows from the fact that the functions $w^{0}, w^{1}$ and $\bar{w}^{0}$ defined in (4-3), (4-4) and (4-5) are exponentially decaying.

Lemma 6.1. There exists a constant $c>0$, not depending on $\tau \in I$ or on $n$ and satisfying

$$
\begin{array}{rlrl}
\left\|\mathscr{L}_{S_{n, \tau}} \Psi_{n}^{T, \vec{a}}\right\|_{\mathscr{C}^{0, \alpha}\left(A_{n, \tau}^{1}\right)} & \leq c e^{-\gamma_{\bar{\tau}, 2} m s_{\bar{\tau}}}, & \left\|\mathscr{L}_{S_{n, \tau}} \Psi_{n}^{T, \vec{a}^{\perp}}\right\|_{\mathscr{C}^{0, \alpha}\left(A_{n, \tau}^{1}\right)} \leq c e^{-\gamma_{\bar{\tau}, 2} m s_{\bar{\tau}}}, \\
\left\|\mathscr{L}_{S_{n, \tau}} \bar{\Psi}_{n}^{T}\right\|_{\mathscr{C}^{0, \alpha}\left(A_{n, \tau}^{0}\right)} \leq c e^{-\gamma_{\tau, 2} n s_{\tau}}, & \left\|\mathscr{L}_{S_{n, \tau}} \bar{\Psi}_{n}^{T}\right\|_{\mathscr{C}^{0, \alpha}\left(A_{n, \tau}^{1}\right)} \leq c e^{-\gamma_{\bar{\tau}, 2} m s_{\bar{\tau}}}, \\
\left\|\mathscr{L}_{S_{n, \tau}} \Psi_{n}^{D}\right\|_{\mathscr{C}^{0, \alpha}\left(A_{n, \tau}^{0}\right)} \leq c n e^{-\gamma_{\tau, 2} n s_{\tau}}, & \left\|\mathscr{L}_{S_{n, \tau}} \Psi_{n}^{D}\right\|_{\left.\mathscr{C}_{0, \alpha} 0, A_{n, \tau}^{1}\right)} \leq c m e^{-\gamma_{\bar{\tau}, 2} m s_{\bar{\tau}}} .
\end{array}
$$

All these estimates are easy to obtain, except maybe the last two, where one has to take into account that the functions under consideration grow "linearly" along the pieces parametrized by $Y_{n, \tau}^{0}$ and $Z_{n, \tau}^{0}$ and hence are not bounded independently of $n$ but rather by a constant times $n$ in $A_{n, \tau}^{0}$ and $A_{n, \tau}^{1}$.

Mapping properties. We define weighted spaces on $S_{n, \tau}$. Roughly speaking, to evaluate the norm in this space, we restrict a function to each summand constituting $S_{n, \tau}$ and then evaluate each term using the norm defined in Definition 3.2.

Definition 6.2. Given $r \in \mathbb{N}, \alpha \in(0,1)$ and $\mu \in \mathbb{R}$, we define $\mathscr{C}_{\mu}^{r, \alpha}\left(S_{n, \tau}\right)$ to be the space of functions $w \in \mathscr{C}^{r, \alpha}\left(S_{n, \tau}\right)$ which are invariant under the action of $G_{k}$. This space is endowed with the norm

$$
\|w\|_{\mathscr{C}_{\mu}^{r, \alpha}\left(S_{n, \tau}\right)}:=\left\|\chi_{n, \tau} w\right\|_{\mathscr{D}_{\mu}^{r, \alpha}\left(\Sigma_{n, \tau}\right)}+\left\|\bar{\chi}_{n, \tau} w\right\|_{\mathscr{D}_{\mu}^{r, \alpha}\left(\bar{\Sigma}_{\tau}\right)} .
$$

We also define the 4-dimensional space

$$
\mathscr{K}\left(S_{n, \tau}\right):=\operatorname{Span}\left\{\bar{\Psi}_{n}^{T}, \Psi_{n}^{D}, \Psi_{n}^{T, \vec{a}}, \Psi_{n}^{T, \vec{a}^{\perp}}\right\} .
$$


In the next result we glue together the parametrices for $\mathscr{L}_{\Sigma_{n, \tau}}$ and $\mathscr{L}_{\bar{\Sigma}_{\tau}}$ to obtain a parametrix for $\mathscr{L}_{S_{n, \tau}}$ :

Proposition 6.3. Assume that $\mu \in\left(-\inf \left(\gamma_{\tau, 2}, \gamma_{\bar{\tau}, 2}\right), 0\right)$ is fixed. There exist $n_{0}>0$ and $c>0$ and, for all $n \geq n_{0}, m \in \mathbb{N}$ and $\tau \in I$ for which (5-2) holds, one can find an operator

$$
G_{n, \tau}: \mathscr{C}_{\mu}^{0, \alpha}\left(S_{n, \tau}\right) \rightarrow \mathscr{C}_{\mu}^{2, \alpha}\left(S_{n, \tau}\right) \times \mathscr{K}\left(S_{n, \tau}\right),
$$

such that $w:=\left(w^{1}, w^{2}\right)=G_{n, \tau}(f)$, then $\tilde{w}=w^{1}+w^{2}$ solves $\mathscr{L}_{S_{n, \tau}} \tilde{w}=f$ on $S_{n, \tau}$ and

$$
\|w\|_{\mathscr{C}_{\mu}^{2, \alpha}\left(S_{n, \tau}\right) \times \mathscr{K}\left(S_{n, \tau}\right)} \leq c\|f\|_{\mathscr{C}_{\mu}^{0, \alpha}\left(S_{n, \tau}\right)},
$$

for some constant which does not depend on $\tau \in I$ nor on $n \geq n_{0}$.

It will be convenient to agree that, given $g=\left(g^{1}, g^{2}\right) \in \mathscr{C}_{\mu}^{2, \alpha}\left(S_{n, \tau}\right) \times \mathscr{K}\left(S_{n, \tau}, \tilde{g}\right.$ denotes the function defined by $\tilde{g}=g^{1}+w^{2}$.

Proof. Given a function $g$ defined on $S_{n, \tau}$, it will be convenient to identify the function $\chi_{n, \tau} g$ (resp. $\bar{\chi}_{n, \tau} g$ ) with a function which is defined on $\Sigma_{n, \tau}$ (resp. $\bar{\Sigma}_{\tau}$ ). This identification is done in the natural way on the common parts of the surfaces; on the ends of the corresponding surfaces it is done by identifying $\left(\chi_{n, \tau} g\right) \circ Z_{n, \tau}^{0}$ with $\left(\chi_{n, \tau} g\right) \circ X_{n, \tau}^{1}\left(\cdot+m s_{\bar{\tau}}, \cdot\right),\left(\chi_{n, \tau} g\right) \circ Y_{n, \tau}^{0}$ with $\left(\chi_{n, \tau} g\right) \circ X_{n, \tau}^{0}\left(n s_{\bar{\tau}}-\cdot, \cdot\right)$, and so on. Conversely, given a function $g$ defined in $\Sigma_{n, \tau}$ or $\bar{\Sigma}_{\tau}$ we will identify the function $\chi_{n, \tau}^{e} g$ or $\bar{\chi}_{n, \tau}^{e} g$, respectively, with a function defined on $S_{n, \tau}$.

Given $f \in \mathscr{C}_{\mu}^{0, \alpha}\left(S_{n, \tau}\right)$ we want to solve the equation

$$
\mathscr{L}_{S_{n, \tau}} \tilde{w}=f
$$

on $S_{n, \tau}$. We solve

$$
\mathscr{L}_{\Sigma_{n, \tau}} u_{1}=\chi_{n, \tau} f
$$

on $\Sigma_{n, \tau}$ and

$$
\mathscr{L}_{\bar{\Sigma}_{\tau}} u_{2}=\bar{\chi}_{n, \tau} f
$$

on $\bar{\Sigma}_{\tau}$.

The existence of $u_{i}$ follows at once from the analysis made in Section 3 and we have the estimate

$$
\left\|u_{1}\right\|_{\mathscr{D}_{\mu}^{2, \alpha}\left(\Sigma_{n, \tau}\right) \times \mathscr{K}\left(\Sigma_{n, \tau}, n \partial_{\tau} T_{\tau}\right)}+\left\|u_{2}\right\|_{\mathscr{D}_{\mu}^{2, \alpha}\left(\bar{\Sigma}_{\tau}\right) \times \mathscr{K}\left(\bar{\Sigma}_{\tau}\right)} \leq c\|f\|_{\mathscr{C}_{\mu}^{0, \alpha}\left(S_{n, \tau}\right)}
$$

where the constant $c>0$ does not depend on $n$ nor on $\tau \in I$. Observe that the function $u_{1}$ can be decomposed as

$$
u_{1}:=v_{1}+a_{1}\left(\Psi^{D}+n \partial_{\tau} T_{\tau} \Phi^{T, \vec{e}_{2}}\right)+b_{1} \psi^{T, \vec{a}}+c_{1} \psi^{T, \vec{a}^{\perp}},
$$

and the function $u_{2}$ can be decomposed as

$$
u_{2}:=v_{2}+a_{2} \bar{\Psi}^{T} .
$$


This being understood, we define the function $u=\left(u^{1}, u^{2}\right)$ by

$$
u=\left(\chi_{\tau, n}^{e} v_{1}+\chi_{\tau, n}^{e} v_{2} \quad, \quad a_{1} \Psi_{n}^{D}+b_{1} \Psi_{n}^{T, \vec{a}}+c_{1} \Psi_{n}^{T, \vec{a}^{\perp}}+a_{2} \bar{\Psi}_{n}^{T}\right) .
$$

Observe that

$$
\|u\|_{\mathscr{C}_{\mu}^{2, \alpha}\left(S_{n, \tau}\right) \times \mathscr{K}\left(S_{n, \tau}\right)} \leq c\|f\|_{\mathscr{C}_{\mu}^{0, \alpha}\left(S_{n, \tau}\right)}
$$

for some constant which does not depend on $n$ nor on $\tau \in I$. We claim that

$$
\left\|\mathscr{L}_{S_{n, \tau}} \tilde{u}-f\right\|_{\mathscr{C}_{\mu}^{0, \alpha}\left(S_{n, \tau}\right)} \leq c\left(n e^{-\gamma_{\tau, 2} n s_{\tau}}+e^{2 \mu n s_{\tau}}+n e^{-\gamma_{\bar{\tau}, 2} m s_{\bar{\tau}}}+e^{2 \mu m s_{\bar{\tau}}}\right)\|f\|_{\mathscr{C}_{\delta}^{0, \alpha}\left(S_{n, \tau}\right)} \text {. }
$$

Since our problem is invariant under the action of $G_{k}$, it is enough to evaluate this quantity on $Y_{n, \tau}^{0}\left(\left(-n s_{\tau}, n s_{\tau}\right) \times S^{1}\right)$ and on $Z_{n, \tau}^{0}\left(\left(-m s_{\bar{\tau}}, m s_{\bar{\tau}}\right) \times S^{1}\right)$. We focus our attention on the estimate of $\mathscr{L}_{S_{n, \tau}} \tilde{u}-f$ on $Y_{n, \tau}^{0}\left(\left(-n s_{\tau}, 0\right) \times S^{1}\right)$, since the estimates on the other parts can be obtained similarly.

In $Y_{n, \tau}^{0}\left(\left(-n s_{\tau}+2,-1\right) \times S^{1}\right)$, all the elements of $\mathscr{K}\left(S_{n, \tau}\right)$ are pieces of Jacobi fields in the sense that, for all $U \in \mathscr{K}\left(S_{n, \tau}\right)$,

$$
\mathscr{L}_{S_{n, \tau} U}=0
$$

in this set. Therefore, $\mathscr{L}_{S_{n, \tau}} \tilde{u}-f=\mathscr{L}_{S_{n, \tau}}\left(v_{1}+v_{2}\right)-f=\mathscr{L}_{S_{n, \tau}} v_{1}$ in this set. We now use the fact that $Y_{n, \tau}^{0}\left(\left(-n s_{\tau}+2,-1\right) \times S^{1}\right)$ can be considered as a normal graph over $E_{n}^{0}$ for some function bounded and whose derivatives are bounded by a constant times $e^{-\gamma_{\tau, 2}\left(s+n s_{\tau}\right)}$ in $\left(-n s_{\tau}, 0\right) \times S^{1}$. In particular, this implies that

$$
\mathscr{L}_{S_{n, \tau}}-\mathscr{L}_{\Sigma_{n, \tau}}
$$

is a second order partial differential operator whose coefficients as well as their derivatives are bounded by a constant times $e^{-\gamma_{\tau, 2}\left(s+n s_{\tau}\right)}$ in $\left(-n s_{\tau}, 0\right) \times S^{1}$. Since $\mathscr{L}_{\Sigma_{n, \tau}} v_{1}=0$ in this set, we conclude that

$$
\begin{aligned}
\left\|e^{-\mu\left(s+n s_{\tau}\right)}\left(\mathscr{L}_{S_{n, \tau}} \tilde{u}-f\right)\right\|_{\mathscr{C}^{0, \alpha}\left(Y_{n, \tau}^{0}\left(\left(-n s_{\tau}+1,-1\right) \times S^{1}\right)\right)} & \\
& \leq c\left(e^{2 n \mu s_{\tau}}+e^{-\gamma_{\tau, 2} n s_{\tau}}\right)\|f\|_{\mathscr{C}_{\delta}^{0, \alpha}\left(S_{n, \tau}\right.} .
\end{aligned}
$$

In $Y_{n, \tau}^{0}\left(\left(-n s_{\tau}+1,-n s_{\tau}+2\right) \times S^{1}\right)$, we obtain, using similar arguments and taking into account the influence of the cutoff function $\chi_{n, \tau}^{e}$, we have

$$
\left\|\mathscr{L}_{S_{n, \tau}} \tilde{u}-f\right\|_{\mathscr{C}^{0, \alpha}\left(Y_{n, \tau}^{0}\left(\left(-n s_{\tau}+1,-n s_{\tau}+2\right) \times S^{1}\right)\right)} \leq c e^{2 n \mu s_{\tau}}\|f\|_{\mathscr{C}_{\delta}^{0, \alpha}\left(S_{n, \tau}\right)},
$$

and in $Y_{n, \tau}^{0}\left((-1,0) \times S^{1}\right)$ we obtain, using similar arguments together with the result of Lemma 6.1,

$$
\left\|e^{-\mu n s_{\tau}}\left(\mathscr{L}_{S_{n, \tau}} \tilde{u}-f\right)\right\|_{\left.\mathscr{C}^{0, \alpha}\left(Y_{n, \tau}^{0}(-1,0) \times S^{1}\right)\right)} \leq c n e^{-\gamma_{\tau, 2} n s_{\tau}}\|f\|_{\mathscr{C}_{\delta}^{0, \alpha}\left(S_{n, \tau}\right)} .
$$

So far, we have produced a linear operator

$$
G_{n, \tau}^{0}: \mathscr{C}_{\mu}^{0, \alpha}\left(S_{n, \tau}\right) \rightarrow \mathscr{C}_{\mu}^{2, \alpha}\left(S_{n, \tau}\right) \oplus \mathscr{K}\left(S_{n, \tau}\right),
$$


defined by $G_{n, \tau}^{0}(f):=u$, which is uniformly bounded (with respect to $n \in \mathbb{N}$ and $\tau \in I)$ and which satisfies

$$
\left\|\mathscr{L}_{S_{n, \tau}} \circ \tilde{G}_{n, \tau}^{0}-I\right\| \leq c\left(n e^{-\gamma_{\tau, 2} n s_{\tau}}+e^{2 \mu n s_{\tau}}+n e^{-\gamma_{\bar{\tau}, 2} m s_{\bar{\tau}}}+e^{2 \mu m s_{\bar{\tau}}}\right)
$$

for some constant independent of $n \in \mathbb{N}$ and $\tau \in I$, where $\tilde{G}_{n, \tau}^{0}(f)$ is the function associated to $G_{n, \tau}^{0}$. The result then follows from a simple perturbation argument, provided $n$ is chosen large enough.

\section{Perturbation of $S_{n, \tau}$}

We define the functions

$$
\Lambda(\tau):=\frac{1}{T_{\bar{\tau}}}\left(\sin (\pi / k)\left(d_{0}\left(\tau, \alpha_{k}\right)+\bar{d}_{0}(\tau)\right)-d^{1}\left(\tau, \alpha_{k}\right)\right)
$$

and

$$
\Gamma(\tau):=2 \sin (\pi / k) \frac{T_{\tau}}{T_{\bar{\tau}}} .
$$

Recall that $\tau$ and $\bar{\tau}$ are related through (5-1). We now prove the main result of the paper:

Theorem 7.1. There exists $n_{0}>0$ such that, for all $n \geq n_{0}$ and all $\tau \in I$ satisfying

$$
\Lambda(\tau)+n \Gamma(\tau) \in \mathbb{N},
$$

the surface $S_{n, \tau}$ can be perturbed into a constant mean curvature 1 surface.

Proof. We consider surfaces that can be written as a normal graph over $S_{n, \tau}$, for some function $w \in \mathscr{C}_{\mu}^{2, \alpha}\left(S_{n, \tau}\right) \times \mathscr{K}\left(S_{n, \tau}\right)$. The equation which guarantees that this surface has constant mean curvature equal to 1 can be written as

$$
\mathscr{L}_{S_{n, \tau}} \tilde{w}+\mathscr{2}_{n, \tau}(\tilde{w})=1-H_{S_{n, \tau}},
$$

where $\tilde{w}$ is the function associated to $w$, where $\mathscr{L}_{S_{n, \tau}}$ is the Jacobi operator about $S_{n, \tau}, H_{S_{n, \tau}}$ is the mean curvature of $S_{n, \tau}$ and $\mathscr{2}_{n, \tau}$ collects all the nonlinear terms. It should be clear from the construction of $S_{n, \tau}$ that, given $r \in \mathbb{N}$, there exists $c_{r}>0$ (independent of $\tau \in I$ and of $n \in \mathbb{N}$ ) such that the pointwise bound

$$
\left|2_{n, \tau}\left(w_{2}\right)-2_{n, \tau}\left(w_{1}\right)\right|_{\mathscr{C}^{r}} \leq c_{r}\left(\left|w_{2}\right|_{\mathscr{C}^{r+2}}+\left|w_{1}\right|_{\mathscr{C}^{r+2}}\right)\left|w_{2}-w_{1}\right|_{\mathscr{C}^{r+2}}
$$

holds provided $\left|w_{1}\right|_{\mathscr{C}^{1}}+\left|w_{2}\right|_{\mathscr{C}^{1}} \leq 1$, where

$$
|w|_{\mathscr{C}} r=\sum_{j=0}^{r}\left|\nabla^{j} w\right|
$$

and partial derivatives are computed using the vector fields $\partial_{s}$ and $\partial_{\theta}$ along the pieces of $S_{n, \tau}$ parametrized by $Y_{n, \tau}^{\ell}$ and $Z_{n, \tau}^{\ell}$ and using a fixed set of vector fields (independent of $n$ ) away from these pieces. 
We fix $\mu \in\left(-\inf \left(\gamma_{\tau, 2}, \gamma_{\bar{\tau}, 2}\right), 0\right)$. Using the result of Proposition 6.3, our problem reduces to finding a fixed point for:

$$
F_{n, \tau}(w)=G_{n, \tau}\left(1-H_{S_{n, r}}-2_{n, \tau}(\tilde{w})\right) .
$$

which belongs to $\mathscr{C}_{\mu}^{2, \alpha}\left(S_{n, \tau}\right) \times \mathscr{K}_{S_{n, \tau}}$. It follows from the result of Lemma 5.1 that

$$
\left\|1-H_{S_{n, \tau}}\right\|_{\mathscr{C}_{\mu}^{0, \alpha}\left(S_{n, \tau}\right)} \leq c\left(e^{-\left(\gamma_{\tau, 2}+\mu\right) n s_{\tau}}+e^{-\left(\gamma_{\bar{t}, 2}+\mu\right) m s_{\bar{\tau}}}\right) .
$$

We set

$$
\rho_{n, \tau}:=\left(e^{-\left(\gamma_{\tau, 2}+\mu\right) n s_{\tau}}+e^{-\left(\gamma_{\bar{\tau}, 2}+\mu\right) m s_{\bar{\tau}}}\right) .
$$

Applying the result of Proposition 6.3, we conclude that

$$
\left\|G_{n, \tau}\left(1-H_{S_{n, \tau}}\right)\right\|_{\mathscr{C}_{\mu}^{2, \alpha}\left(S_{n, \tau}\right) \times \mathscr{K}\left(S_{n, \tau}\right)} \leq \bar{c} \rho_{n, \tau}
$$

for some constant $\bar{c}>0$ which does not depend on $\tau \in I$ nor on $n \in \mathbb{N}$, for which (7-1) holds.

Now, it follows from (7-3) that there exists a constant $c>0$, not depending on $\tau \in I$ or on $n \in \mathbb{N}$, such that

$$
\begin{aligned}
& \left\|\mathscr{2}_{n, \tau}\left(\tilde{w}_{2}\right)-\mathscr{2}_{n, \tau}\left(\tilde{w}_{1}\right)\right\|_{\mathscr{C}_{\mu}^{0, \alpha}\left(S_{n, \tau}\right)} \\
& \quad \leq c n^{2}\left(e^{-\mu n s_{\tau}}+e^{-\mu m s_{\bar{\tau}}}\right) \rho_{n, \tau}\left\|w_{2}-w_{1}\right\|_{\mathscr{C}_{\mu}^{2, \alpha}\left(S_{n, \tau}\right) \otimes \mathscr{K}\left(S_{n, \tau}\right)},
\end{aligned}
$$

provided $\left\|w_{2}\right\|_{\mathscr{C}_{\mu}^{2, \alpha}\left(S_{n, \tau}\right) \times \mathscr{K}\left(S_{n, \tau}\right)}+\left\|w_{1}\right\|_{\mathscr{C}_{\mu}^{2, \alpha}\left(S_{n, \tau}\right) \times \mathscr{K}\left(S_{n, \tau}\right)} \leq 2 \bar{c} \rho_{n, \tau}$. The $n^{2}$ which appears in this estimate arises from the fact that the element $\Psi_{n, \tau}^{D}$ of $\mathscr{K}\left(S_{n, \tau}\right)$ is not bounded uniformly in $n$, but is bounded, as well as its derivatives, by a constant (independent of $\tau$ and $n$ ) times $n$.

We choose $\mu<0$ close enough to 0 so that

$$
\lim _{n \rightarrow+\infty} n^{2}\left(e^{-\mu n s_{\tau}}+e^{-\mu m s_{\bar{\tau}}}\right) \rho_{n, \tau}=0
$$

uniformly for $\tau \in I$. (Recall that $n$ and $m$ are related by (7-1); hence they are commensurable, and in particular there exists $c>0$, independent of $\tau \in I$, such that $n \leq c m$ and $m \leq c n$.)

That $F_{n, \tau}$, for $n$ large enough, has a fixed point in the ball of radius $2 \bar{c} \rho_{n, \tau}$ in $\mathscr{C}_{\mu}^{2, \alpha}\left(S_{n, \tau}\right) \oplus \mathscr{K}\left(S_{n, \tau}\right)$ follows directly from (7-5) and (7-6).

The surfaces we have obtained are immersed, compact surfaces with genus $k$ (these surfaces are not embedded since the Type- 1 elements which have been used for their construction are never embedded). The surfaces obtained for different values of $\tau$ and $n$ satisfying (7-1) are geometrically different (noncongruent) provided $n_{0}$ is chosen large enough. Hence, the set of solutions of (7-1) give a local picture of the set of compact constant mean curvature surfaces of genus $k$ with symmetry group $G_{k}$. 
Finally, observe that Lemma 2.3, together with relation (5-1) between $\tau$ and $\bar{\tau}$, implies that $\partial_{\tau}\left(T_{\tau} / T_{\bar{\tau}}\right)>0$. In particular, one can define the change of variable

$$
\zeta:=\Gamma(\tau)
$$

and the function $\Xi(\zeta)=\Lambda \circ \Gamma^{-1}(\zeta)$ so that constant mean curvature surfaces can be constructed provided $\zeta \in O:=\Gamma(I)$ and $n \geq n_{0}$ satisfy

$$
\Xi(\zeta)+\zeta n \in \mathbb{N}
$$

\section{References}

[Cosín and Ros 2001] C. Cosín and A. Ros, "A Plateau problem at infinity for properly immersed minimal surfaces with finite total curvature", Indiana University Math. J. 50:2 (2001), 847-879. MR 2003d:53013 Zbl 1041.53004

[Delaunay 1841] C. Delaunay, "Sur la surface de revolution dont la courbure moyenne est constante", J. de Mathématiques 6 (1841), 309-320.

[Große-Brauckmann 1993] K. Große-Brauckmann, "New surfaces of constant mean curvature", Math. Z. 214:4 (1993), 527-565. MR 94k:53012 Zbl 0806.53005

[Große-Brauckmann 2000] K. Grosse-Brauckmann, R. B. Kusner, and J. M. Sullivan, "Constant mean curvature surfaces with three ends", Proc. Natl. Acad. Sci. USA 97:26 (2000), 14067-14068. MR 2001j:53009 Zbl 0980.53011

[Große-Brauckmann and Kusner 1999] K. Große-Brauckmann and R. B. Kusner, "Embedded constant mean curvature surfaces with special symmetry", Manuscripta Math. 99:1 (1999), 135-150. MR 2000e:53010 Zbl 0970.53007

[Große-Brauckmann et al. 2003] K. Große-Brauckmann, R. B. Kusner, and J. M. Sullivan, "Triunduloids: embedded constant mean curvature surfaces with three ends and genus zero", J. Reine Angew. Math. 564 (2003), 35-61. MR 2005a:53009 Zbl 1058.53005

[Jleli 2004] M. Jleli, Constant mean curvature hypersurfaces, Ph.D. thesis, Université de Paris 12, 2004.

[Jorge and Meeks 1983] L. P. Jorge and W. H. Meeks, III, "The topology of complete minimal surfaces of finite total Gaussian curvature”, Topology 22:2 (1983), 203-221. MR 84d:53006 Zbl 0517.53006

[Kapouleas 1990] N. Kapouleas, "Complete constant mean curvature surfaces in Euclidean threespace”, Ann. of Math. (2) 131:2 (1990), 239-330. MR 93a:53007a Zbl 0699.53007

[Kapouleas 1991] N. Kapouleas, "Compact constant mean curvature surfaces in Euclidean threespace”, J. Differential Geom. 33:3 (1991), 683-715. MR 93a:53007b Zbl 0727.53063

[Kapouleas 1995] N. Kapouleas, "Constant mean curvature surfaces constructed by fusing Wente tori”, Invent. Math. 119:3 (1995), 443-518. MR 95m:53008 Zbl 0840.53005

[Korevaar et al. 1989] N. J. Korevaar, R. Kusner, and B. Solomon, "The structure of complete embedded surfaces with constant mean curvature", J. Differential Geom. 30:2 (1989), 465-503. MR 90g:53011 Zbl 0726.53007

[Korevaar et al. 2004] N. Korevaar, R. Kusner, and J. Ratzkin, "On the nondegeneracy of constant mean curvature surfaces", preprint, 2004. math.DG/0407153

[Kusner et al. 1996] R. Kusner, R. Mazzeo, and D. Pollack, "The moduli space of complete embedded constant mean curvature surfaces”, Geom. Funct. Anal. 6:1 (1996), 120-137. MR 97b:58022 Zbl 0966.58005 
[Mazzeo and Pacard 2001] R. Mazzeo and F. Pacard, "Constant mean curvature surfaces with Delaunay ends”, Comm. Anal. Geom. 9:1 (2001), 169-237. MR 2002b:53005 Zbl 1005.53006

[Mazzeo et al. 2001] R. Mazzeo, F. Pacard, and D. Pollack, "Connected sums of constant mean curvature surfaces in Euclidean 3 space", J. Reine Angew. Math. 536 (2001), 115-165. MR 2002d: 53020 Zbl 0972.53010

[Mazzeo et al. 2005] R. Mazzeo, F. Pacard, and D. Pollack, "The conformal theory of Alexandrov embedded constant mean curvature surfaces in $\mathbb{R}^{3}$,, pp. 525-559 in Global theory of minimal surfaces, edited by D. Hoffman, Clay Mathematics Proceedings 2, Amer. Math. Soc., Providence, 2005.

[Pinkall and Sterling 1989] U. Pinkall and I. Sterling, "On the classification of constant mean curvature tori”, Ann. of Math. (2) 130:2 (1989), 407-451. MR 91b:53009 Zbl 0683.53053

[Ratzkin 2001] J. Ratzkin, An end-to-end gluing construction for surfaces of constant mean curvature, Ph.D. thesis, University of Washington, Seattle, 2001.

[Wente 1986] H. C. Wente, "Counterexample to a conjecture of H. Hopf", Pacific J. Math. 121:1 (1986), 193-243. MR 87d:53013 Zbl 0586.53003

Received October 16, 2003. Revised October 1, 2004.

\section{MOHAMED JLELI}

ECOlE Supérieure des SCIENCES ET TEChNiques DE Tunis (ESSTT)

5 AVENUE TAHA-HUSSEIN

TUNIS BP 56

BAB MENARA 1008

TUNISIA

Email: jleli@caramail.com

FRANK PACARD

UNIVERSITÉ PARIS 12

61 Avenue du GÉNÉral de Gaulle

94010 CRÉTEIL

FRANCE

and

INSTITUT UNIVERSITAIRE DE FRANCE

pacard@univ-paris12.fr 